\title{
Article \\ Characteristics of SlCML39, a Tomato Calmodulin-like Gene, and Its Negative Role in High Temperature Tolerance of Arabidopsis thaliana during Germination and Seedling Growth
}

\author{
Haidong Ding $1,2, * \mathbb{C}$, Ying Qian ${ }^{2}$, Yifang Fang ${ }^{2}$, Yurong $\mathrm{Ji}^{2}$, Jiarong Sheng ${ }^{2}$ and Cailin Ge ${ }^{1,2, *}$ \\ 1 Joint International Research Laboratory of Agriculture and Agri-Product Safety of Ministry of Education of \\ China, Yangzhou University, Yangzhou 225009, China \\ 2 College of Bioscience and Biotechnology, Yangzhou University, Yangzhou 225009, China; \\ 18852721947@163.com (Y.Q.); fyf010102@163.com (Y.F.); jyr0806@163.com (Y.J.); myyjjfb@163.com (J.S.) \\ * Correspondence: hdding@yzu.edu.cn (H.D.); clge@yzu.edu.cn (C.G.); Tel./Fax: +86-514-8797-9204
}

\section{check for}

updates

Citation: Ding, H.; Qian, Y.; Fang, Y.; Ji, Y.; Sheng, J.; Ge, C. Characteristics of SlCML39, a Tomato

Calmodulin-like Gene, and Its Negative Role in High Temperature Tolerance of Arabidopsis thaliana during Germination and Seedling Growth. Int. J. Mol. Sci. 2021, 22, 11479. https://doi.org/10.3390/ ijms222111479

Academic Editor: Hye Ryun Woo

Received: 26 September 2021

Accepted: 21 October 2021

Published: 25 October 2021

Publisher's Note: MDPI stays neutral with regard to jurisdictional claims in published maps and institutional affiliations.

Copyright: (c) 2021 by the authors. Licensee MDPI, Basel, Switzerland. This article is an open access article distributed under the terms and conditions of the Creative Commons Attribution (CC BY) license (https:// creativecommons.org/licenses/by/ $4.0 /)$

\begin{abstract}
Calmodulin-like (CML) proteins are primary calcium sensors and function in plant growth and response to stress stimuli. However, so far, the function of plant CML proteins, including tomato, is still unclear. Previously, it was found that a tomato (Solanum lycopersicum) CML, here named SlCML39, was significantly induced by high temperature (HT) at transcription level, but its biological function is scarce. In this study, the characteristics of SICML39 and its role in HT tolerance were studied. SlCML39 encodes a protein of 201 amino acids containing four EF hand motifs. Many cis-acting elements related to plant stress and hormone response appear in the promoter regions of SlCML39. SlCML39 is mainly expressed in the root, stem, and leaf and can be regulated by HT, cold, drought, and salt stresses as well as ABA and $\mathrm{H}_{2} \mathrm{O}_{2}$. Furthermore, heterologous overexpression of SlCML39 reduces HT tolerance in Arabidopsis thaliana at the germination and seedling growth stages. To better understand the molecular mechanism of SICML39, the downstream gene network regulated by SlCML39 under HT was analyzed by RNA-Seq. Interestingly, we found that many genes involved in stress responses as well as ABA signal pathway are down-regulated in the transgenic seedlings under HT stress, such as KIN1, RD29B, RD26, and MAP3K18. Collectively, these data indicate that SlCML39 acts as an important negative regulator in response to HT stress, which might be mediated by the ABA signal pathway.
\end{abstract}

Keywords: Arabidopsis thaliana; calmodulin-like protein; high temperature; RNA sequencing; SlCML39; tomato

\section{Introduction}

Calcium $\left(\mathrm{Ca}^{2+}\right)$ is a key secondary messenger in eukaryotic cells which is involved in plant growth, development, and stress responses [1]. The $\mathrm{Ca}^{2+}$ signal is recognized by unique $\mathrm{Ca}^{2+}$ sensors, including calmodulin (CaM), calmodulin-like protein (CML), calcineurin B-like (CBL), and calcium-dependent protein kinase (CDPK) [1,2]. All these proteins have a conserved $\mathrm{Ca}^{2+}$ binding motif- "EF hand"-which binds to $\mathrm{Ca}^{2+}$ to cause conformational changes, followed by changes in activity to regulate downstream targets, thereby transmitting $\mathrm{Ca}^{2+}$ signals [3]. CaM is the most widely studied $\mathrm{Ca}^{2+}$ sensor. CMLs are closely related to CaMs, but their appearance is different from CaM, which has four EF hands. CMLs have one to six EF hands [4]. According to the existing genome database, many CMLs are identified in plants [5], such as 50 CMLs in Arabidopsis thaliana [6], 46 in Medicago truncatula [2], and 168 in Brassica napus [7]. Despite so many CML genes, the functions of most plant CMLs are not well studied [8].

To date, CML genes have been found to be involved in plant growth, development, cell metabolism, and stress tolerance. AtCML24 and AtCML25 mutants strongly affect Arabidopsis pollen germination and tube growth $[9,10]$. AtCML39 participates in light signal 
transduction and promotes the establishment of Arabidopsis seedlings [11]. AtCML42 transduces $\mathrm{Ca}^{2+}$ signal downstream by interacting with kinesin-interacting $\mathrm{Ca}^{2+}$-binding protein (KIC), then regulates the cell branches of hair [12] and also coordinates responses to Spodoptera herbivory [13]. CML41 regulates plasmodesmatal closure during plant immune responses [14]. CML36 regulates the activity of the $\mathrm{Ca}^{2+}$ ATPase ACA8 [15]. AtCML9 knockout mutants exhibit stronger salt and drought tolerance through high accumulation of amino acids [16]. In Arabidopsis, a new rice CML gene OsMSR2 endows plants with drought and salt tolerance through an ABA-mediated pathway [17]. GsCML27 is involved in plant responses to bicarbonate, salt, and osmotic stresses [18]. CML20 negatively modulates ABA signals in guard cells and drought tolerance [19]. MpCML40 overexpression strongly increases Arabidopsis salt tolerance [20]. In all, these studies suggest that CMLs likely interpret $\mathrm{Ca}^{2+}$ signals during development and stress responses.

To date, CML function analyses mainly come from Arabidopsis. In tomato, genomewide identification and characterization of CML genes in two genetic backgrounds (Solanum lycopersicum and Solanum pennellii) were performed [21,22]. A total of 45 and 52 CML genes were identified in Solanum pennellii and Solanum lycopersicum, respectively. So far, however, there are only three reports on tomato CML function. A tomato CML (APR134) is involved in plant-Pseudomonas syringae interactions [23]. ShCML44 overexpression improves tomato tolerance to cold, drought, and salinity stress [21]. Recently, S1CML37 has been shown to interact with proteasome maturation factor 1 (SIUMP1) to improve chill tolerance of tomato fruits [8]. The functions of other CMLs in tomato still need to be improved.

In a previous report, we found that a tomato (Solanum lycopersicum) CML gene (Solyc11g071740) was significantly induced by high temperature (HT) at transcription level using RNA-seq analysis of HT-responsive genes in tomato leaves [24]. However, the function of this gene responding to HT is unknown. In this study, the CML gene, named SlCML39, was characterized. SlCML39 was mainly expressed in the root, stem, and leaf and could be regulated by different stresses. Moreover, heterologous overexpression of SlCML39 remarkably reduced Arabidopsis tolerance to HT stress. The transcriptome by RNA-seq analysis further showed that many genes related with stress response as well as the ABA signal pathway were down-regulated in the transgenic seedlings under HT stress. Taken together, our results indicate that as an important negative regulator, SlCML39 plays a role in HT stress response, which might be mediated by the ABA signal pathway.

\section{Results}

\subsection{SlCML39 Is Attractive Enough under HT Stress}

Previously, we found that a tomato (Solanum lycopersicum) CML gene (Solyc11g071740) was significantly induced by HT at the transcription level using RNA-seq analysis of tomato HT-responsive genes [24]. Munir et al. [25] identified 52 CML genes in tomato (Solanum lycopersicum) using genome analysis and Solyc11g071740 was named as SICML39. Therefore, we continue to use this name here. Interestingly, HT-induced SlCML39 expression was mediated by SIMPK1 (Figure 1). Our previous data have shown that tomato SIMPK1 (mitogen-activated protein kinase 1) is a negative regulator in HT tolerance [26]. Here, HT-induced SICML39 expression was further increased in SIMPK1-overexpressing plants, but this inductive effect disappeared in SlMPK1-RNA interference plants under HT stress (Figure 1B,C), suggesting that SlCML39 functions downstream of SIMPK1, and that SIMPK1 positively regulates the expression of SlCML39 under HT stress. The discovery of SlCML39 is exciting because its function can be studied to further elaborate the response mechanism of SIMPK1 to HT stress. 

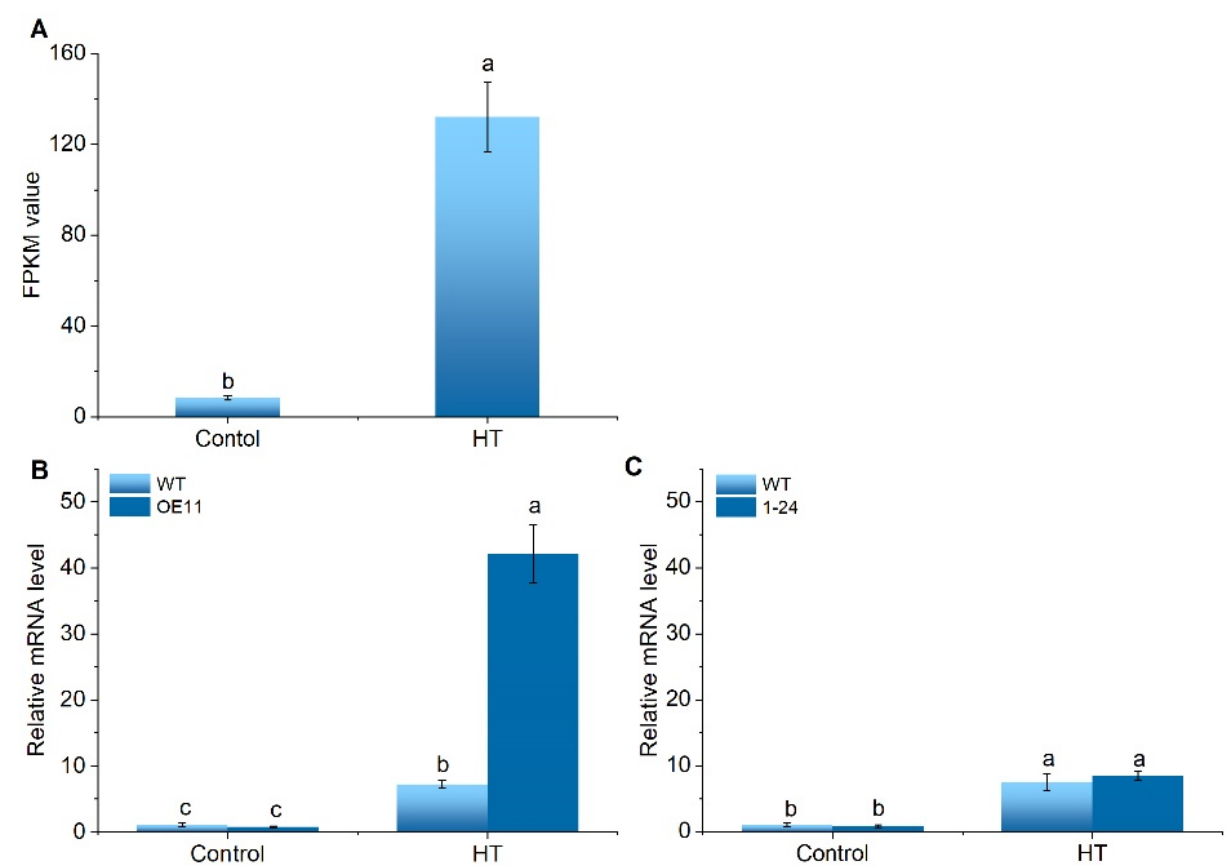

Figure 1. High temperature-induced SICML39 expression is regulated by SIMPK1 under high temperature (HT). (A) The transcript level of SICML39 was induced by HT using RNA-seq analysis of HT-responsive genes in tomato leaves [24]. (B) qRT-PCR analysis of SlCML39 expression in wild-type (WT) and SIMPK1-overexpressing line OE11 under HT. (C) qRT-PCR analysis of SICML39 expression in WT and SIMPK1-RNA interference line 1-24 under HT. The UBI gene was used as an internal standard. Total RNAs were extracted from WT, OE11, or 1-24 leaves under control $\left(25^{\circ} \mathrm{C}\right)$ and HT $\left(42{ }^{\circ} \mathrm{C}\right)$ for $4 \mathrm{~h}$. The bar represents the mean $\pm \mathrm{SD}$ values of three replicates $(n=3)$. According to the LSD test, the statistical differences between samples are marked with different letters $(p<0.05)$.

\subsection{Characterization of SICML39}

The CDS of SlCML39 contains 606 bp open reading frame (ORF), which is on chromosome 11 without introns (Figure 2A). The cDNA encodes a protein of 201 amino acids, of which the predicted molecular mass is $22,118.77 \mathrm{Da}$ and the theoretical $\mathrm{pI}$ is 4.79 . Ten conserved motifs were predicted using the MEME tool (Figure 2B). To investigate the potential regulatory functions of SlCML39, the cis regulatory elements in the $2000 \mathrm{bp}$ fragment upstream of the Transcription Start Site (TSS) were analyzed using the PlantCARE tool (Figure 2C, Table S2). The results showed that in addition to the core cis-elements such as TATA box and CAAT box, many types of cis-elements were found, including plant growth regulation, stress response, hormone response, and light response elements (Figure 2C, Table S2). For stress-responsive cis-elements, MYB and MYC were the most abundant elements. ARE (anaerobic response element), STRE (stress-response promoter element), as-1 (oxidative stress-responsive element), WUN-motif (wound-responsive element), and W-box (WRKY binding sites) were also present. For hormone-responsive cis-elements, four MeJA-responsive elements (CGTCA-motif and TGACG-motif), four estrogen response elements (ERE), one SA-responsive element (TCA-element), two GA-responsive elements (TATC-box, GARE-moti), and one auxin-responsive element (TGA-element) were detected in the promoter of SlCML39. The results here suggest that SlCML39 might play a role in the transcriptional control of tomato growth, stress, and hormone responses. 
A

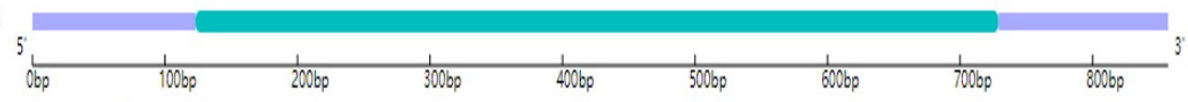

Legend:

$\mathrm{CDS}$ UTR

B $p$-value $9.95 e-109$

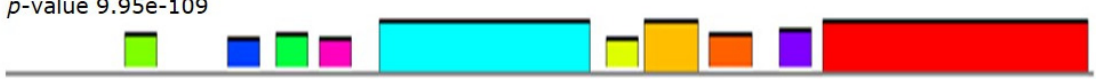

C
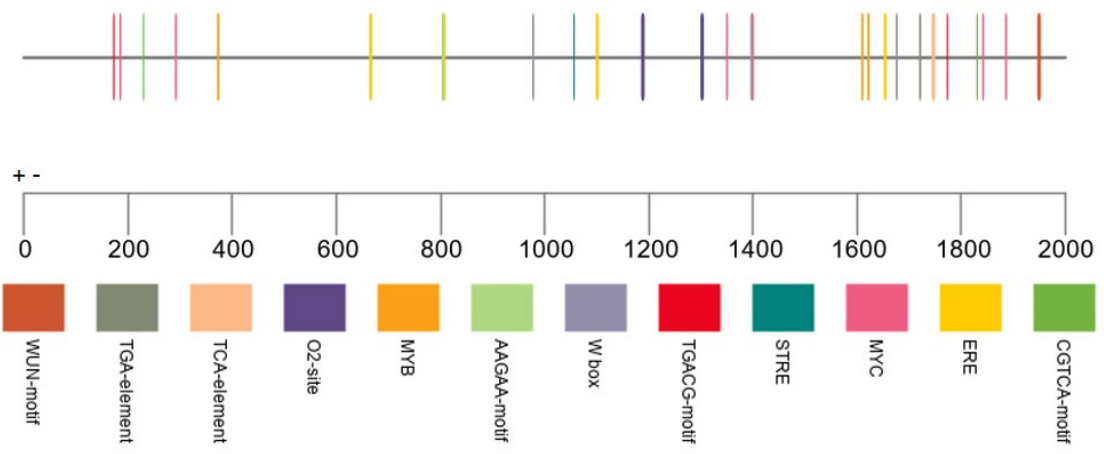

Figure 2. Characters of SlCML39. (A) SlCML39 in the tomato genome. The SlCML39 gene structure was visualized using GSDS 2.0. (B) The SICML39 motif was analyzed using the MEME web server. (C) Cis-element analysis of SlCML39 promoter. The cis-element of SlCML39 promoter was analyzed by PlantCARE database. All elements are shown in Supplementary Table S1.

To characterize the function of SlCML39, a phylogenetic tree was constructed according to the amino acid sequence of SICML39 and all Arabidopsis thaliana AtCMLs (Figure S1), or some other plant species using MUSCLE/PhyML/TreeDyn programs (Figure 3A). The phylogenetic tree revealed that SICML39 closely clustered with Soltu.DM.11G024600, which is because both tomato and potato belong to Solanaceae, so the homologous genes have the highest similarity. In addition to this protein, SICML39 is also similar to some other members of the subfamily, including AtCML37, AtCML38, AtCML39, OSCML19, and ZmPHB47.K022800.1.p, which indicate that SICML39 might have a similar function with these proteins. Recently, SlCML37 confers improved tolerance to tomato fruit chilling stress [8]. However, SICML39 and SICML37 are not the same protein (Figure 3A). A comparison of the protein sequences between SICML39 and five homology proteins of other plants is presented in Figure 3B. SICML39 shares a high degree of similarity to OsCML19 (56.74\%), AtCML37 (56.03\%), AtCML38 (54.48\%), AtCML39 (54.031\%), and AtCML41 (41.14\%). Based on the InterPro protein families database (EF hand domain IPR002048) and Zhang et al.'s [20] analysis, the conservative functional domains identified in these CMLs are $\mathrm{Ca}^{2+}$-binding EF hands.

\subsection{Expression Profiles of SlCML39}

To study the spatial expression pattern of SICML39 in tomato, the ePLANT data for SICML39 expression was obtained from the tomato ePLANT browser (bar.utoronto.ca). Unfortunately, the expression of chip data was low and not accurate enough. Therefore, SlCML39 expression levels in different organs were determined by qRT-PCR. The results displayed that SICML39 was expressed in most of the organs. The expression of SlCML39 was the highest in root, followed by stem, leaf, and red fruit (Figure 4), indicating the organ specificity of SlCML39 expression in tomato. 
A

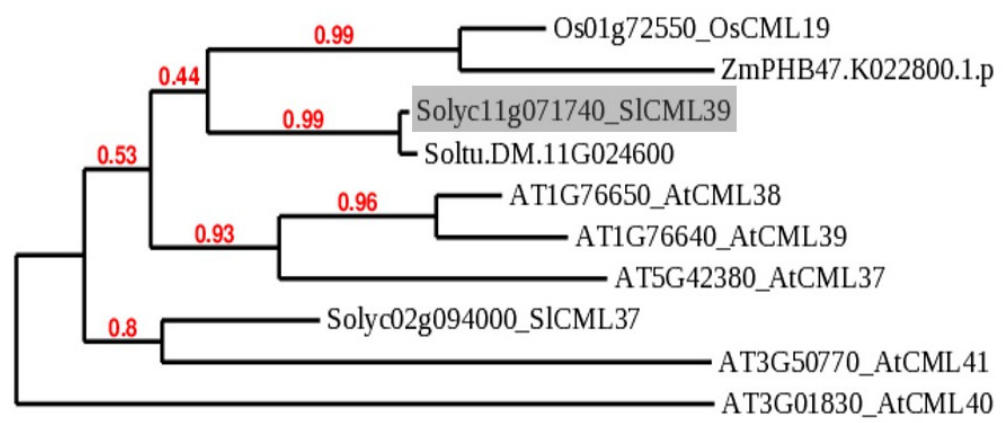

B 0.4

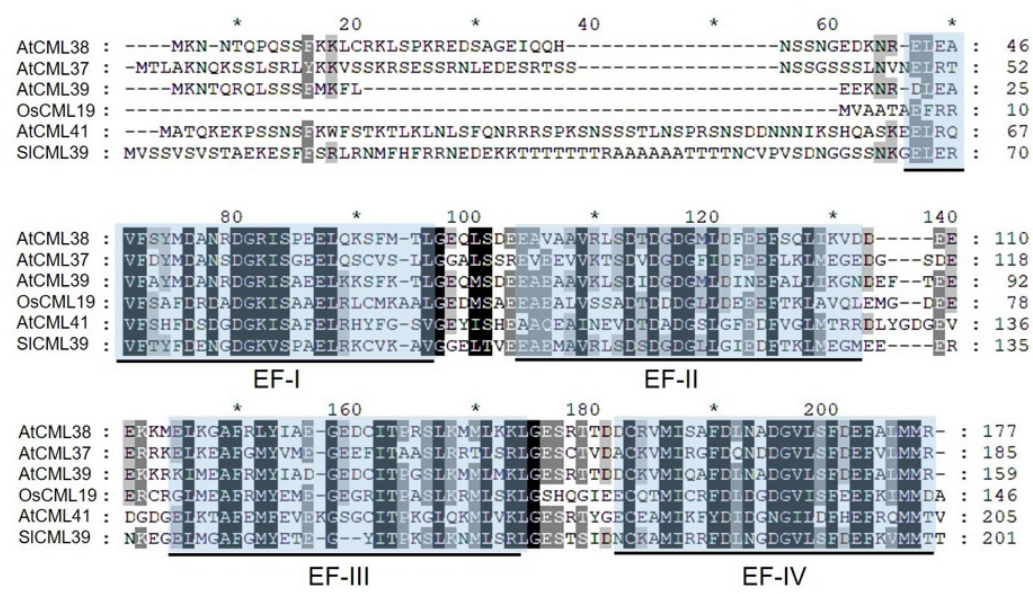

Figure 3. Phylogenetic tree and multiple sequence alignment of SICML39 and its closely related CML proteins from other plant species. (A) The phylogenetic tree was constructed using MUSCLE/PhyML programs. The following protein sequences are included in the analysis: Arabidopsis (AT5G42380, AtCML37; AT1G76650, AtCML38; AT1G76640, AtCML39; AT3G01830, AtCML40; AT3G50770, AtCML41); Oryza sativa (Os01G72550, OsCML19); Zea mays (ZmPHB47.K022800.1.p); Solanum lycopersicum (Solyc11g071740, SlCML39); Solanum tuberosum (Soltu.DM.11G024600). SlCML39 is marked in gray. (B) Multiple sequence alignment of SICML39 sequence with homologous CMLs from Arabidopsis and rice. Sequences were aligned by MUSCLE and shown by GeneDoc software. The EF hand motifs are underlined and the highly conserved amino acids in EF hand motifs are highlighted by the blue color.

To explore the potential roles of SlCML39 in stress responses, we then performed an analysis of the expression pattern of SlCML39 under drought, salt, HT, cold, ABA, and $\mathrm{H}_{2} \mathrm{O}_{2}$ (Figure 5). Under 20\% PEG6000 treatment, the expressions of SlCML39 observably increased at different time points and reached the highest levels at $24 \mathrm{~h}$; moreover, $100 \mathrm{mM}$ $\mathrm{NaCl}$ stress could significantly induce SlCML39 expression at the different time points, except for $6 \mathrm{~h}$. After ABA treatment, the expression of SlCML39 reached the highest levels at $6 \mathrm{~h}$, then showed a gradual reduction. The SlCML39 expression level was induced by $10 \mathrm{mM} \mathrm{H}_{2} \mathrm{O}_{2}$ at 3,6 , and $12 \mathrm{~h}$, with about 12 -fold accumulated transcripts at $3 \mathrm{~h}$. The expression of SlCML39 increased rapidly, reached the highest levels at $1 \mathrm{~h}$, then showed a gradual reduction under cold stress $\left(4^{\circ} \mathrm{C}\right)$; $\mathrm{HT}\left(42^{\circ} \mathrm{C}\right)$ significantly induced SICML39 expression, reaching the maximum at $3 \mathrm{~h}$. These results indicate that SICML39 might be involved in response to multiple abiotic stresses (Figure 5). 



Figure 4. Expression levels of SlCML39 in different organs. (A) ePlant of microarray data of SlCML39 expression from bar.utoronto.ca. (B) The organ-specific expression of SlCML39 was detected in the root, stem, leaf, flower, green fruit, and red fruit by qRT-PCR. Total RNA was extracted from different organs. The UBI gene was used as an internal control. The bar represents the mean $\pm \mathrm{SD}$ values of three replicates $(n=3)$. According to the LSD test, the statistical differences between samples are marked with different letters $(p<0.05)$.
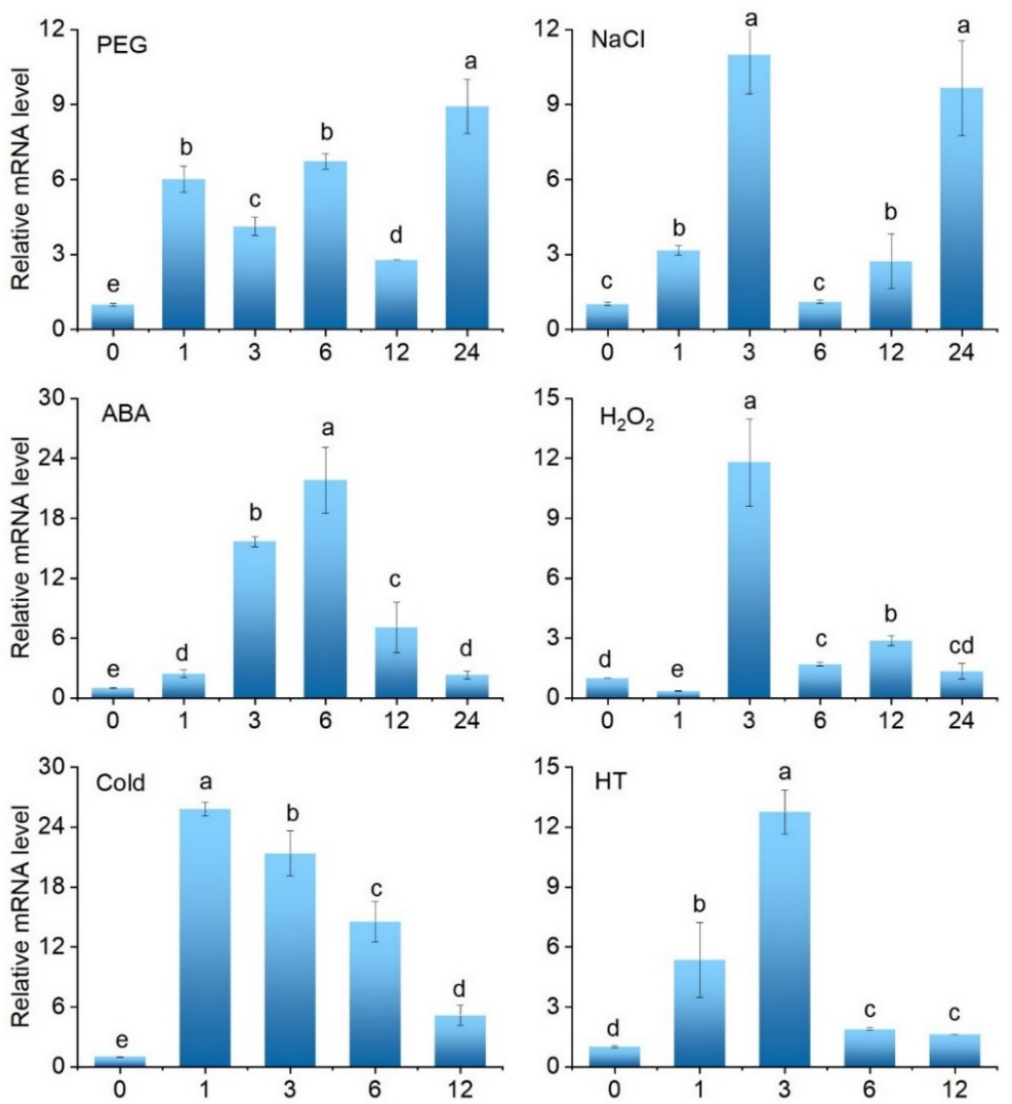

Figure 5. Expression levels of SlCML39 under different treatments. Tomato seedlings were treated with $\mathrm{H}_{2} \mathrm{O}, 20 \%$ PEG6000, $100 \mathrm{mM} \mathrm{NaCl}, 100 \mathrm{uM} \mathrm{ABA}, 10 \mathrm{mM} \mathrm{H}_{2} \mathrm{O}_{2}$, cold $\left(4^{\circ} \mathrm{C}\right)$, or high-temperature conditions (HT, $42{ }^{\circ} \mathrm{C}$ ) for $1,3,6,12$, and/or $24 \mathrm{~h}$. Total RNA was extracted from leaves and relative mRNA levels were determined by qRT-PCR using UBI gene as an internal control. The fold change was normalized relative to the level of $\mathrm{H}_{2} \mathrm{O}$ treatment. The bar represents the mean $\pm \mathrm{SD}$ values of three replicates $(n=3)$. According to the LSD test, the statistical differences between samples are marked with different letters $(p<0.05)$. 


\subsection{SlCML39 Negatively Regulated Seed Germination in Arabidopsis under HT Stress}

SICML39 has a higher expression level at the transcription under HT stress (Figures 1 and 5). HT-induced SlCML39 expression is regulated by SIMPK1 (Figure 1), which has been found involved in thermotolerance [26]. We speculated that SlCML39 might be involved in HT response. To prove this, the transgenic Arabidopsis plants overexpressing SlCML39 were obtained (Figure 6A). Two homozygous lines (OE4-8, OE7-9) were obtained and used to evaluate thermotolerance. The expression level of SlCML39 in OE4-8 and OE7-9 was verified by qRT-PCR analysis (Figure 6B). First, we conducted a plate germination test to determine the HT tolerance of Arabidopsis lines OE4-8 and OE7-9 and WT (Figure 6C-F). Seeds of Arabidopsis were immersed at $52{ }^{\circ} \mathrm{C}$ for 15 or $25 \mathrm{~min}$, then sown on $1 / 2 \mathrm{MS}$ medium. The germination rate and posted-growth phenotype were observed within 8 days. Under normal conditions (seeds without HT pretreatment), OE4-8 and OE7-9 plants showed similar germination and post-geminated growth with WT, indicating that SlCML39 does not affect seed germination (Figure $6 \mathrm{C}, \mathrm{F}$ ) and early seedling growth (Figure 6D,E). After HT pretreatment, however, OE4-8 and OE7-9 plants displayed lower germination rates, lower biomass of post-germinated seedlings, and fewer seedlings with open and green leaves than WT. In all, during imbibition, transgenic SlCML39-overexpressing seeds exhibited a thermosensitive phenotype.



Figure 6. Overexpression of SICML39 increases the sensitivity of Arabidopsis thaliana seed germination to high temperature (HT). (A) Schematic diagram of the SICML39 expression vector. The coding region of SICML39 was cloned in the expression vector pBI121. (B) The mRNA levels of SICML39 in WT and two SICML39-overexpressing transgenic lines, OE4-8 and OE7-9, using qRT-PCR. ACTIN gene was used as an internal control. (C) Seed germination of WT and SICML39-overexpressing lines on $1 / 2$ MS medium after HT. Dry seeds were immersed at $52{ }^{\circ} \mathrm{C}$ for 15 (T1) and $25 \mathrm{~min}$ (T2), then sterilized on the surface before sowing. The germination rate within 8 days was calculated. The phenotype under light for 4 days is shown. (D) Post-germinated growth of WT and SlCML39-overexpressing lines on 1/2 MS medium after HT for 14 days. (E) The fresh weight of (D). (F) The germination rates of $(C)$. The bar represents the mean \pm SD values of three replicates $(n=3)$. According to the LSD test, the statistical differences between samples are marked with different letters $(p<0.05)$. 


\subsection{SlCML39 Overexpression Decreased Arabidopsis Seedling Tolerance to HT Stress}

HT-induced inhibition of seedling growth assay has been used to identify plant HT tolerance [26]. Consistent with the seed germination type, there was no obvious difference between WT and transgenic line OE4-8 and OE7-9 plants during the growth stage under the standard conditions (Figure 7), suggesting that SlCML39 does not affect seedling development. After exposure to HT stress, all plants showed burning symptoms caused by HT. However, SlCML39-overexpressing plants had a more serious HT damage phenotype, lower chlorophyll content, and lower fresh weight when compared with WT (Figure 7). With the extension of HT treatment time, the damage phenotype caused by HT aggravated, which was more serious in transgenic plants than in WT. Together, the results demonstrated that SICML39 plays a negative role in HT tolerance.
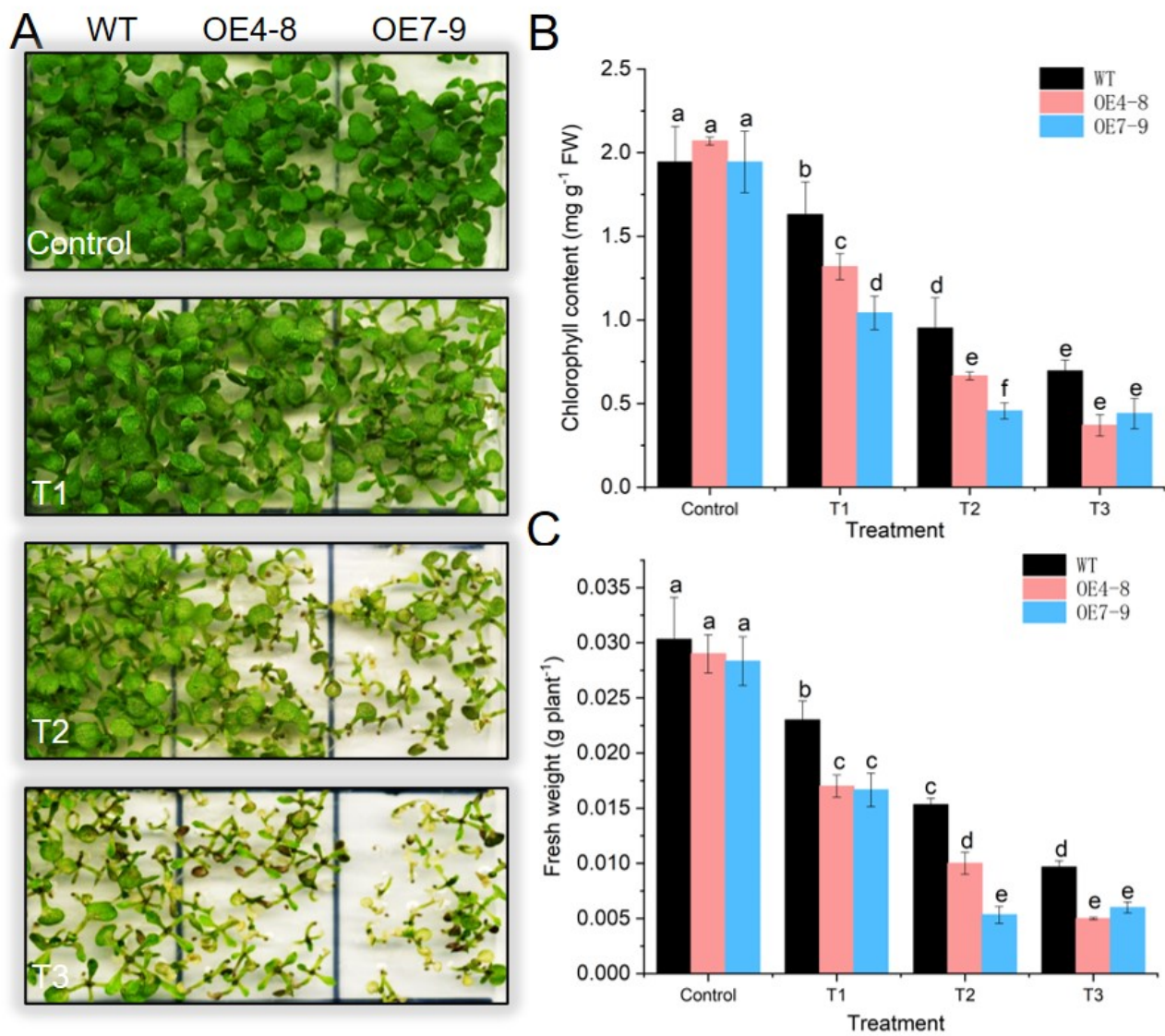

Figure 7. Overexpression of SlCML39 increases the sensitivity of seedling growth to high temperature (HT). (A) Phenotype of seedling growth of SlCML39-overexpressing lines (OE4-8 and OE7-9) after HT. After germination on $1 / 2 \mathrm{MS}$ medium for 7 days, the seedlings on plates were exposed to $45^{\circ} \mathrm{C}$ for $0 \mathrm{~min}$ (control), $45 \mathrm{~min}$ (T1), $60 \mathrm{~min}$ (T2), and $90 \mathrm{~min}$ (T3), followed by recovery under standard conditions $\left(23^{\circ} \mathrm{C}\right)$ for 7 days. (B) Chlorophyll content of (A). (C) Plant fresh weight of (A). The bar represents the mean $\pm \mathrm{SD}$ values of three replicates $(n=3)$. According to the LSD test, the statistical differences between samples are marked with different letters $(p<0.05)$.

\subsection{RNA-Seq of SlCML39-Regulating Gene Network under HT Stress}

In order to fully provide the regulatory network of SlCML39 in HT response in Arabidopsis and gain insight into the mechanism of SlCML39, we conducted an RNA-seq analysis to screen DEGs-regulated by SICML39. According to Lu's experimental design of analyzing HT response by RNA-seq [27], we performed an RNA-seq analysis of OE7-9 and WT under HT stress using BGISEQ-500 platform [28]. Six cDNA libraries (three replicates per library) were set up from OE7-9 and WT plants under HT stress, and the boxplots revealed that the overall range and distribution of FPKM values of these samples are 
consistent (Figure S2), indicating that the RNA-seq data in this study have reproducibility, high quality, and reliability. Additionally, we analyzed the expression profiles among the two groups in the pairwise comparisons. DEGs were analyzed with a 2.0 -fold change as a selection threshold. Gene expressions with a $\geq 2.0$-fold or $\leq 0.5$-fold change were recorded for significant up- or down-regulation, respectively; an adjusted $p$-value (padj) $\leq 0.05$ was applied to both screenings.

Through rigorous screening for three biological repetitions, we screened a total of 38 DEGs (Table 1), of which 28 were down-regulated and 10 were up-regulated in SlCML39-overexpressing line OE7-9. According to GO annotation, all DEGs are divided into 34 groups, including 19 biological processes, 6 cellular components, and 9 molecular functions (Figure S2B). In biological processes, the DEGs are mainly involved in cellular processes, metabolic processes, stimulus response, biological regulation, and the regulation of biological processes. The 20 top GO enrichment shows that a large number of stress-responsive DEGs are modulated by SICML39, such as "response to stimulation (19)", "response to hormone (11)", and "response to ABA (11)" (Figures 8 and S2C). In terms of molecular function, there are two categories: "binding" and "catalytic activity". To screen the pathways regulated by SICML39, the KEGG pathway annotation was performed, and MAPK signal pathway is the only pathway with a $q$-value $<0.05$. To further confirm the results, qRT-PCR analysis of six DEGs was used to validate the data. Six genes displayed similar expression trends at the transcript level (Figure S3). The results indicate that SlCML39-regulated DEGs might be responsible for the decreased HT tolerance in SlCML39-overexpressing plants.

A

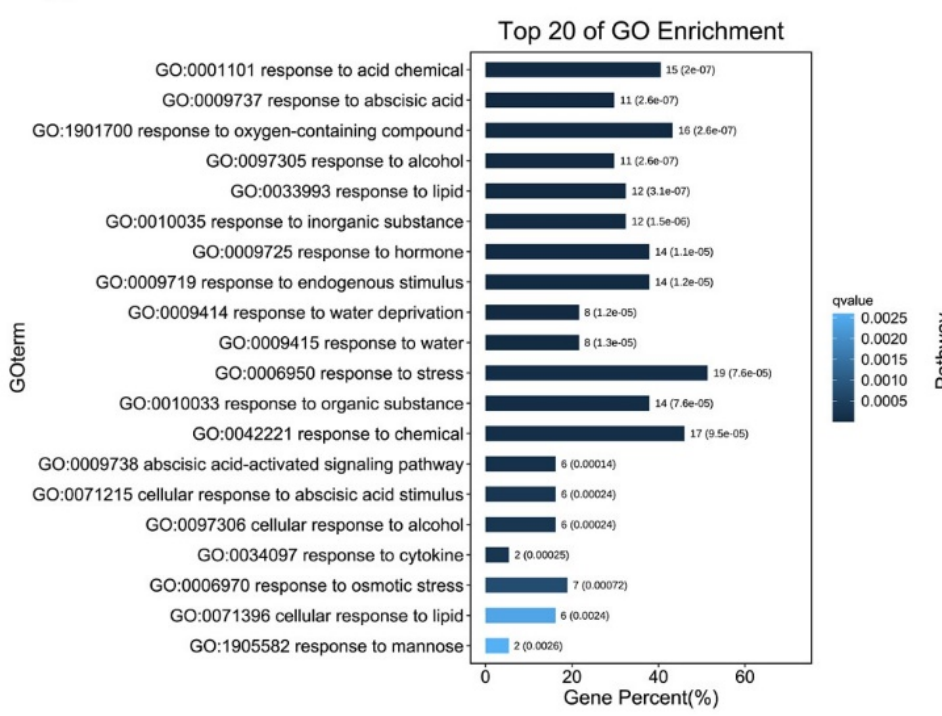

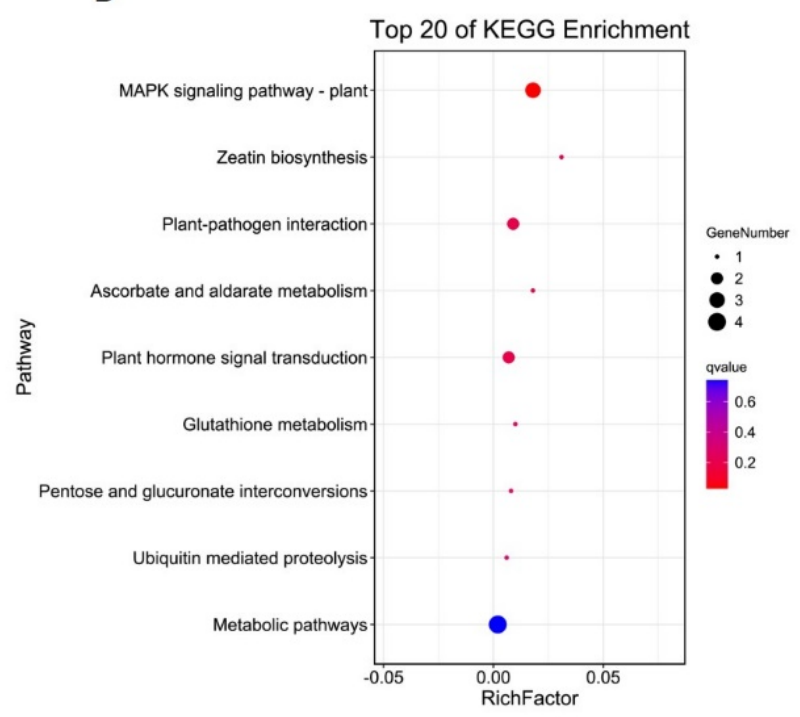

Figure 8. GO term and KEGG enrichment analyses of DEGs. (A) Top of 20 GO term enrichment. The vertical coordinates are the enriched GO terms, and the horizontal coordinates are the numbers of the DEGs. (B) KEGG enrichment analysis of DEGs. The vertical coordinates are the enriched pathways, and the horizontal coordinates are the rich factors. The size of each point represents the number of DEGs in the pathway, and the point color represents the $q$-value. 
Table 1. Different expressed genes in 35S: SICML39 compared to wild-type Arabidopsis thaliana under high temperature ( adj $<0.05$; | fold change $\mid>2$ ).

\begin{tabular}{|c|c|c|c|c|}
\hline GeneID & Fold Change & padj & Symbol & Description \\
\hline AT3G09940** & -1.96 & 0.000 & MDAR3 & Monodehydroascorbate reductase 3 \\
\hline AT4G21830 **** & -1.92 & 0.000 & MSRB7 & Methionine sulfoxide reductase B7 \\
\hline AT1G02920 & -1.55 & 0.005 & GSTF7 & Glutathione S-transferase 7 \\
\hline AT1G53903 * & -1.47 & 0.015 & & Linoleate 9S-lipoxygenase- 4 protein \\
\hline AT1G53885 * & -1.47 & 0.015 & & Linoleate 9S-lipoxygenase-4 protein \\
\hline AT3G50770 * & -1.39 & 0.010 & CML41 & Calmodulin-like 41 \\
\hline AT3G44006 * & -1.37 & 0.038 & & unknown protein \\
\hline AT3G15450*,** & -1.36 & 0.000 & & Aluminum-induced protein \\
\hline AT5G52300 *,** & -1.36 & 0.029 & RD29B & Low-temperature 65 \\
\hline AT1G24793* & -1.35 & 0.019 & AtLpxC1 & lipid X C1 \\
\hline AT1G64110 ** & -1.34 & 0.010 & DAA1 & DUO1-activated ATPase 1 \\
\hline AT2G43820 ** & -1.34 & 0.000 & SAGT1 & UDP-glucosyltransferase 74F2 \\
\hline AT4G12530 *** & -1.32 & 0.012 & AZI7 & Bifunctional inhibitor \\
\hline AT4G27410 **** & -1.24 & 0.047 & RD26 & Responsive to desiccation 26 \\
\hline AT3G44860 *,** & -1.22 & 0.001 & FAMT & Farnesoic acid carboxyl-O-methyltransferase \\
\hline AT5G59220 **** & -1.21 & 0.000 & SAG113 & Highly ABA-induced PP2C gene 1/HAI1 \\
\hline AT3G63060*,** & -1.21 & 0.020 & EDL3 & EID1-like 3 \\
\hline AT5G02020 *,** & -1.19 & 0.000 & SIS & Salt-Induced Serine-rich \\
\hline AT3G48360 *,** & -1.18 & 0.039 & ATBT2 & BTB and TAZ domain protein 2 \\
\hline AT1G05100 **** & -1.15 & 0.021 & MAPKKK18 & MAP kinase kinase kinase 18 \\
\hline AT1G23390 **** & -1.12 & 0.000 & KFB & A kelch domain-containing F-box protein \\
\hline AT3G21220 & -1.11 & 0.000 & MKK5 & MAP kinase kinase 5 \\
\hline AT5G15960 **** & -1.10 & 0.001 & KIN1 & Stress-responsive protein (KIN1) \\
\hline AT5G15500 *,** & -1.07 & 0.000 & & Ankyrin repeat family protein \\
\hline AT5G67480 *,** & -1.07 & 0.001 & ATBT4 & BTB and TAZ domain protein 4 \\
\hline AT3G $27250 * * * *$ & -1.06 & 0.009 & DIL4 & ABA-induced transcription repressor \\
\hline AT5G22920 **** & -1.01 & 0.004 & AtRZPF34 & RING-type Zinc finger protein \\
\hline AT1G24260* & -1.00 & 0.008 & SEP3 & MADs box transcription factor \\
\hline AT3G29030 **** & 1.01 & 0.000 & ATEXP5 & Expansin A5 \\
\hline AT3G56360* & 1.02 & 0.000 & & Hypothetical protein \\
\hline AT1G35260* & 1.04 & 0.033 & MLP165 & MLP-like protein 165 \\
\hline AT3G27650 * & 1.13 & 0.024 & LBD25 & LOB domain-containing protein 25 \\
\hline AT2G43870 * & 1.21 & 0.001 & & Pectin lyase-like superfamily protein \\
\hline AT2G41510 * & 1.21 & 0.010 & ATCKX1 & Cytokinin oxidase \\
\hline AT2G38530 * & 1.24 & 0.000 & ATLTPI-5 & Lipid transfer protein 2 \\
\hline AT1G13740 *,** & 1.26 & 0.001 & AFP2 & ABI five binding protein 2 \\
\hline AT1G44800 **** & 1.30 & 0.000 & SIAR1 & Siliques Are Red 1 \\
\hline AT2G32990 **** & 1.30 & 0.000 & GH9B8 & Glycosyl hydrolase 9B8 \\
\hline
\end{tabular}

Fold change indicates values from the three independent replicates of up/down-regulation in 35S: SlCML39 line OE7-9 compared with wild-type (Col-0) under high temperature. **** Indicates that the gene expression was induced by high temperature and ABA, respectively, based on the GO biological process (response to ABA) or the studies of high temperature-induced transcriptomics in Arabidopsis (Tables S3 and S4). All 38 gene expression data are in Table S5.

\subsection{SlCML39 Negatively Regulates Stress/ABA-Responsive Genes under HT Stress}

Because 38 DEGs were mainly clustered into "response to stress" and "response to ABA" and SlCML39 expression was also induced by HT, we screened the genes of HT response and ABA response. Chao et al. [29] mapped heat-responsive genes to an ABA-responsive gene pool to identify whether the heat-responsive genes were also ABAresponsive genes. In this study, although these genes were not clustered into the heat shock response, we mapped 38 HT-responsive DEGs list to three HT-responsive gene pools [30-32] and two ABA-responsive gene pools [33,34] to discern whether these DEGs were HT or ABA-responsive genes. Combined with GO analysis and Arabidopsis eFP Browse database, 33 and 22 DEGs were identified to be involved in HT and ABA response, respectively (Table 1 and Table S3). Moreover, 21 of the 33 DEGs (63.6\%) that responded to HT were able to respond to ABA (Table 1). Interestingly, the 28 down-regulated DEGs 
in this study were compared to the study in 2016 [33] and it was found that 16 of them were ABA-responsive genes such as RD29B, DAA1, RD26, FAMT, SAG113, EDL3, SIS, MAPKKK18, KIN1, and DIL4 (Table S4). Many DEGs have been found in HT tolerance. For example, the transcription levels of RD29B, RD26, FAMT, SAG113, EDL3, KIN1, and DIL4 were induced by HT, of which the RD26, SAG113, KIN1, and DIL4 expressions in heat-sensitive $g c n 5$ Arabidopsis were lower than that in WT [31]. Here, the visualization of RNA-seq readings of these genes confirmed that the expressions of these genes are down-regulated in SlCML39-overexpressing plants compared to WT after application of HT. RD29B, RD26, SAGT1, SAG113, KIN1, DIL4, and AFP2 are ABA-responsive genes, which play an important role in ABA signal and stress responses [35,36]. ABA is also involved in acquired thermotolerance [37,38]. Except for AFP2, the expression levels of these DEGs under HT stress were also decreased in SlCML39-overexpressing plants compared with WT. In all, there were 21 DEGs responding to HT and ABA simultaneously, indicating that the negative regulation of HT tolerance by SlCML39 might be mediated by ABA signal. Additionally, three DEGs involved in the MAPK signal pathway (HAI1, $M A P K K K 18$, and MKK5) were down-regulated in SlCML39 overexpression plants. MAPK cascade is involved in response to HT stress [26,39]. ROS-induced redox signal acts as an important regulator of various stress responses [40]. Interestingly, the expression levels of ATMDAR3, ATMSRB7, and GSTF7 decreased in the SlCML39-overexpressing plants under HT compared with WT (Table 1).

To evaluating the reliability of results of RNA-Seq, the relative expressions of six typical stress/ABA-responsive DEGs in the SlCML39 overexpression plant and WT treated with or without HT were validated by qRT-PCR (Figure S3). The expression pattern of the selected stress-related genes by qRT-PCR mostly agreed with RNA-Seq data, although the absolute fold changes of the two methods were different, indicating that the results obtained by RNA-seq analysis are credible.

\subsection{SlCML39 Is Involved in ABA-Mediated Seed Germination}

To further confirm the role of SlCML39 in ABA signal, we tested the ABA sensitivity of SlCML39-overexpressing plants (OE4-8 and OE7-9) relative to WT plants at the germination and post-germination stages under ABA treatment. SlCML39 ectopic expression lines exhibited similar seed germination with WT. However, under 1.0 and $1.5 \mu \mathrm{m} \mathrm{ABA}$ treatment, transgenic lines displayed lower seed germination rates (Figure 9A) than WT. The higher the ABA concentration, the stronger the inhibition. The hypersensitivity of SlCML39-overexpressing plants to ABA was also observed in the post-germination stage. Under normal conditions, there was no significant difference in cotyledon opening rate and greening rate between transgenic plants and WT (Figure 9B,C). Under 1.0 $\mu \mathrm{m}$ ABA, there was no obvious difference. However, under $1.5 \mu \mathrm{m}$ ABA supplement, the cotyledon opening and greening rates of OE4-8 and OE7-9 were inhibited more severely in transgenic lines than in WT (Figure 9B,C). The results demonstrated that SlCML39 confers plants more sensitive to ABA-inhibited germination and post-geminated growth, suggesting that SlCML39 is involved in the ABA signal. 
A

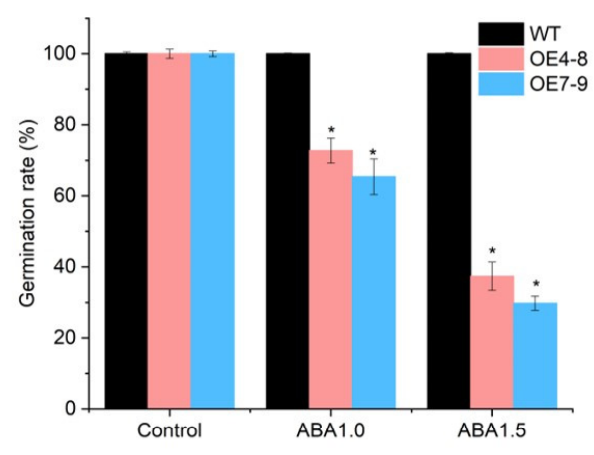

B

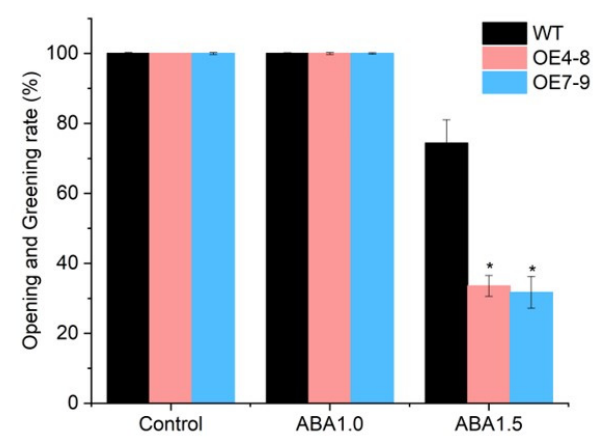

C

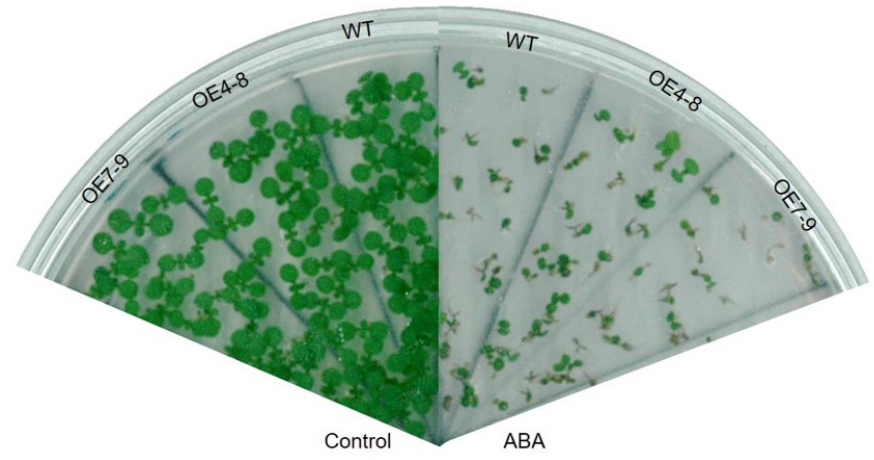

Figure 9. Overexpression of SlCML39 increases Arabidopsis thaliana sensitivity to ABA. (A) The germination rate of SlCML39-overexpressing lines (OE4-8 and OE7-9) and wild-type (WT) Arabidopsis seeds on 1/2 MS medium supplementing 1.0 and $1.5 \mu \mathrm{m}$ ABA. Germination was recorded daily up to 5 days. (B) Seedlings with open and green leaves were recorded at 10 days. (C) The photographs were taken at 10 days. The bar represents the mean \pm SD values of three replicates $(n=3) .{ }^{*} p<0.05$ by Student's $t$-test.

\section{Discussion}

\subsection{Homologous CMLs of SlCML39}

The CMLs are a unique group of EF hand proteins in plants, which bind to $\mathrm{Ca}^{2+}$ and regulate downstream signal transduction [3]. In Arabidopsis thaliana, 50 AtCMLs were identified according to Arabidopsis genome sequence, which are divided into eight groups [6]. In this study, SICML39 belongs to $\mathrm{Ca}^{2+}$-binding proteins with four EF hands, which are clustered in group IV of AtCMLs [6]. SlCML39 has the highest sequence similarity with AtCML37, AtCML38, and AtCML39. These three CMLs act as sensors in $\mathrm{Ca}^{2+}$-mediated growth, development, and stress response [41]. AtCML37 loss-of-function mutants showed increased sensitivity to herbivores and decreased tolerance to drought stress [42]. CML38 is a core hypoxia response $\mathrm{Ca}^{2+}$ sensor protein and serves as a potential $\mathrm{Ca}^{2+}$ signal target during flooding stress response [43]. As an AtRALF1-interacting partner, CML38 participates in the inhibitory effect of AtRALF1 on root growth [44]. CML38 specifically interacts with PEP1 RECEPTOR 2 (PEPR2) and negatively regulates Arabidopsis root elongation under low nitrate [45]. As a Ca ${ }^{2+}$ sensor, CML39 is involved in the transduction of light signals that promote seedling establishment [11]. CML39 plays a key role in seed and fruit development and hormone-mediated seed dormancy [46]. Recently, it was found that SICML37 interacts with proteasome maturation factor SIUMP1 and confers improved chilling tolerance in tomato fruit [8]. All these results imply that SICML39 might have similar functions, which requires further functional studies.

\subsection{SlCML39 Expression Is Induced by HT as well as Other Stresses}

We speculated that SlCML39 could function under HT stress because of its higher expression induced by HT (Figure 1). To date, there is no report on the functional research of CMLs in response to HT, while some CML genes in plants can respond to HT stress. It is 
reported that Arabidopsis AtCML12 (TCH3) and AtCML24 (TCH2) expressions are highly induced by heat stress [47]. AtCML44 is strongly induced by heat [6]. Two BnaCML44 genes (BnaA06g15280D and BnaC05g16860D) are enhanced by heat [7]. In this study, HT $\left(42^{\circ} \mathrm{C}\right)$ significantly induced SlCML39 expression, reaching the maximum in $3 \mathrm{~h}$ (Figure 5). In addition, SlCML39 expression was regulated by drought, salt, HT, and cold stresses, as well as exogenous $\mathrm{ABA}$ and $\mathrm{H}_{2} \mathrm{O}_{2}$, similar to the previous studies. For example, the expressions of AtCML37, AtCML38, and AtCML39 genes are induced by a variety of stimuli such as salt and drought stress [41]. AtCML9 expression in young seedlings is rapidly induced by cold, salinity, and dehydration treatments [16]. Expression of 32 CML genes in wild-growing grapevine shows that it can respond to drought, salt, heat, and cold [48]. Through analysis of upstream promoter sequences with the promoter tool PlantCARE, a variety of different boxes correlated with responses to miscellaneous abiotic stress were found in the SlCML39 promoters (Figure 2C). A variety of stress and hormone response elements were found by promoter element analysis, but there were no heat shock elements (HSE) or ABA-responsive elements (ABRE) (Figure 2C). Heat-induced genes may not always require HSE. Several Dof sequences, a W-box, and an MYC recognition site are present in the heat-responsive region of the MsDREB2C gene [49]. In general, ABRE is necessary for ABA-induced genes. However, other cis-elements that mediate ABA regulated transcription are also known. For example, MYB and MYC binding sites (without any G-ABRE or coupling elements) in the promoter region of $R D 22$ gene are crucial for ABA-dependent drought-induced gene expression [50]. In all, in addition to HT, other stresses could also regulate SlCML39 expression. SlCML39 can be considered as a stress-responsive gene because its expression rapidly changes in response to these stresses. In this study, we focus our research on the function of SlCML39 in HT response.

\subsection{SICML39 Decreased Plant Thermotolerance}

To explore the roles of SlCML39 in tomato under HT stress, SlCML39 was heterologously expressed in Arabidopsis. The transgenic plants overexpressing SlCML39 displayed lower seed germination rates, fewer seedlings with open and green leaves, lower biomass of post-germinated seedlings than WT, and worse seedling growth than WT after HT stress (Figures 6 and 7). It is indicated that SlCML39 plays a negative role in regulating plant thermotolerance. So far, most studies on CMLs show a positive regulation of stress $[8,16,17,20,21]$. For example, OsMSR2 is involved in drought and salt tolerance [17]. Overexpression of $M p C M L 40$ enhances salt tolerance of Arabidopsis [20]. Of course, earlier reports of many systems revealed their negative impacts on plant defense. CML24underexpressing transgenics enhance Arabidopsis tolerance to various salts such as $\mathrm{CoCl}_{2}$, molybdic acid, $\mathrm{ZnSO}_{4}$, and $\mathrm{MgCl}_{2}$ [51]. CML9 is considered as a negative regulator of ABA-dependent salt and drought tolerance [16]. CML42 has a negative effect on ABA biosynthesis upon drought stress [13]. GsCML27 overexpression decreased the salt and osmotic tolerance of Arabidopsis [18]. AtCML20 is a negative regulator in Arabidopsis of ABA-induced stomatal movement and drought tolerance [19]. One explanation for these regulatory mechanisms is the specificity and complexity of CML functions in virous stress signal pathways. However, different regulatory pathways may have common components as crosstalk nodes. Among these regulatory mechanisms, one of the most striking is the CML-mediated ABA signal $[13,16,17,19]$. Interestingly, these HT-intolerant phenotypes observed in the SlCML39-overexpressing plants are attributed to its function of regulating the expression levels of ABA- and HT-responsive genes (Table 1).

\subsection{SlCML39 Is Involved in ABA-Regulated Germination}

It is well known that $\mathrm{Ca}^{2+}$ is a component of the ABA signal pathway and some CML proteins have been proved to be involved in this pathway [17,46,51]. It is well established that $\mathrm{ABA}$ can maintain seed dormancy, arrest seed germination, and prevent seedling growth, and HT can induce ABA biosynthesis and trigger ABA-mediated signal pathways [29,37]. In the current study, HT exerted its inhibitory effect on seed germination 
and post-germinated growth of WT and transgenic plants mostly through ABA production. Under HT stress, the ABA level was up-regulated (Figure S4). However, there was no significant change in the ABA content of transgenic plants compared with WT under HT stress (Figure S4). On the contrary, there was increased sensitivity of the SlCML39overrexpressing transgenics to HT. These results suggest that SlCML39 is not involved in ABA biosynthesis, but rather in the ABA signal pathway under HT. Furthermore, the seed germination experiment regulated by $A B A$ further confirmed this assumption. Previous studies have shown that CMLs function in ABA-regulated seed germination. For instance, during the seed germination and post-germination stages, transgenic OsMSR2 overexpression plants exhibit hypersensitivity to ABA [17]. In this work, Arabidopsisoverexpressing SICML39 increased sensitivity to exogenous ABA (Figure 9). The seeds of transgenics and WT germinated fully under normal conditions. With the increase in $\mathrm{ABA}$ content, the germination rate and cotyledon greening of transgenics seeds were more severely reduced by ABA (Figure 9). This result indicates that SlCML38 acts downstream of the ABA signal, resulting in ABA hypersensitivity during seed germination. Previously, CML24 may act on the downstream signal of ABA perception, perhaps mediating the responses of cells to $\mathrm{ABA}$-induced $\mathrm{Ca}^{2+}$ fluctuations to delay seed germination and seedling growth [51]. The atcml9 mutant germination is hypersensitive to exogenous $\mathrm{ABA}$ and AtCML9 does not play a key role in ABA biosynthesis, but rather in the ABA signal pathway as a negative regulator [16]. The sensitivity of seeds to ABA inhibition of germination is significantly reduced in Arabidopsis cml39 mutant and mutant plants are more likely to be affected than endogenous ABA content [52]. Together, the existing evidence shows that plant CMLs play special functions downstream of the ABA signal.

\subsection{SlCML39 Regulates Stress/ABA-Responsive Genes under HT}

$\mathrm{ABA}$ is a key hormone regulating plant tolerance to abiotic stresses, including temperature change and drought [52]. Application of ABA enhances plant HT tolerance, and the ABA-mediated signal is involved in acquired HT tolerance, as well [37]. Heat stress induces many ABA-responsive genes [29]. To further study the molecular mechanism of the SICML39 negative regulation of HT tolerance, RNA-seq was used to identify the gene network. SlCML39-overexpressing plants showed alterations in the expression of several stress and ABA-responsive genes, many of which were associated with HT stress (Tables 1 and S4). GO enrichment analyses revealed that many DEGs were significantly enriched in the ABA signal pathway and 17 of the 38 DEGs were able to respond to HT and ABA at the same time (Table 1), supporting the notion that Arabidopsis thermotolerance involves the induction of a subset of ABA-responsive genes. Here are two puzzling questions to answer. One is that ABA synthesis-related genes are not found in this study (Table 1). Combined with the above results of SlCML39 involved in seed germination and ABA accumulation level, it is indicated that SlCML39 contributes to HT response in conjunction with the downstream ABA signal, and a role of SlCML39 in regulating stress/ABA-related genes is suggested. Another indication is that HT-related genes of heat shock proteins (HSPs) and heat shock factor (HSFs) are not present. Interestingly, heat stress-triggered hormone responses are independent of the HSP/HSF pathways, indicating the existence of specific links between heat shock perception and hormone responses [29]. Geng et al. [53] revealed that ER stress-related genes are associated with HT tolerance in TabZIP60s overexpressing Arabidopsis by RNA-sequencing analysis, and also few HSP/HSFs are found under HT stress. Our present results imply a specific link between SlCML39 and ABA signal in response to HT stress.

Previous studies have also proved the regulatory relationship between CML and $\mathrm{ABA}$, mainly focusing on drought and salt, and only analyzed some response genes. The atcml9 mutants alter the expression of some stress and ABA-responsive genes as well as defense-related genes [16]. Xu et al. [17] showed that Arabidopsis OsMSR2 confers salt and drought tolerance, accompanied by changes in the expression of stress/ABAresponsive genes such as $R D 29 A, P 5 C S 1$, and ABI3. In the cml20 mutant, the expressions of 
stress genes including $M Y B 2, R A B 18, E R D 10, C O R 47$, and $R D 29 A$ are up-regulated under drought and ABA treatment, suggesting that CML20 plays a role in ABA and drought stress signal pathways [19]. What attracts attention is that this study is the first to study the function of CMLs in HT stress, and proposes that SlCML39-mediated HT tolerance is related to ABA signal pathway. Here, several abiotic stress/ABA-responsive genes such as KIN1, RD26, and RD29B have reduced expression in SlCML39-overexpressing plants (Table 1). Additionally, many genes also play roles in stress tolerance. AtSIS is a salt-induced serine-rich gene and plays a role in salt tolerance, probably by increasing the protective effect of membranes [54]. Exposed to osmotic stress, high salinity, and ABA, EDL3 transcript accumulates and plays an active regulatory role in ABA-dependent signal cascades, regulating germination induction, root growth, greening of etiolated seedlings, and anthocyanin accumulation under drought stress [55]. DAA1 (At1g64110) encodes an AAA+-type ATPase and the response of DAA1 gene knockout mutant to ABA, $\mathrm{NaCl}$, and mannitol is impaired in germination and root growth, indicating stress sensitivity and the role of the DAA1 gene in stress tolerance [56]. OsRZFP34, a homolog AtRZFP34 (At5g22920) in rice, is markedly higher with HT and ABA at the mRNA level in transgenic rice, and OsRZFP34 may increase stomatal opening and transpiration cooling function of rice under HT [57]. SGT1 is a co-chaperone of HSP90 and the response of plants to elevated ambient temperature requires an HSP90-SGT1 chaperone system [58]. Two transcription factors (TFs) were found to be involved in the SICML39-mediated pathway, including RD26/ANAC072 and SEP3/AGL9 (Table 1, Figure 10). RD26 is a NAC TF and functions as a transcriptional activator in ABA-inducible gene expression under abiotic stress in plants [59]. SEP3 is a MADS-box TF and ABA was reported to upregulate the expressions of MADS-box genes. However, the direct relationship between SEP3 and ABA needs to be further studied.



Figure 10. The model of SICML39 as a hub connecting the ABA signal and ABA-mediated heat tolerance. Under heat stress, phosphorylation of the SIMPK1 activates downstream target proteins SISPRH1, resulting in decreased thermotolerance [26]. Heat-induced SlCML39 expression is mediated by SIMPK1, and is involved in negative regulation of heat tolerance. Under heat stress, SlCML39 expression is induced, acts downstream of ABA signal, and inhibits the expressions of $\mathrm{ABA} /$ stress-responsive genes, resulting in decreased thermotolerance. The NAC transcription factor RD26/ANAC072 and MADs box transcription factor SEP3 are involved.

KEGG enrichment analyses showed that three genes, MAPKKK18, MKK5 and SAG113, were significantly enriched in the MAPK signal pathway (Figure 8, Table S3). MKKK18 expression is modulated by ABA and MAPKKK18 positively regulates drought tolerance via downstream MPKK3 in Arabidopsis [60]. MKK5 is involved in AIK1-modulated ABA response through the MKK5-MPK6 kinase cascade and functions in the regulation of ABA on the primary root growth and stomatal response [61]. Inhibition of $M K K 5$ can reduce the 
transmission of ozone-induced signals to MPK3 and MPK6 and increase ozone sensitivity in Arabidopsis [62]. In the current study, the expressions of MAPKKK18 and MKK5 were induced by HT (Table 1). HT-induced SICML39 expression was further increased in SIMPK1-overexpressing plants, but this inductive effect disappeared in SIMPK1-RNA interference plants under HT stress (Figure 1). These results suggest that MAPK cascade is involved in SICML39-regulating HT tolerance, but the specific cascade pathway still needs to be studied.

\section{Materials and Methods}

\subsection{Plant Material Growth}

Tomato (Solanum lycopersicum cv. "OFSN" background: wild-type (WT), SIMPK1overexpressing line OE11, and SIMPK1-RNA interference line 1-24 [26]) and Arabidopsis thaliana (Columbia-0 ecotype background: WT and SlCML39-overexpressing lines (OE4-8 and OE7-9) were used in the present study. For tomato growth, the germinated seeds on the filter paper in the dark were planted in plastic pots containing soil mixture and grown at $25{ }^{\circ} \mathrm{C} / 20{ }^{\circ} \mathrm{C}$ (day/night) and $14 \mathrm{~h}$ of light/10 h of dark with $70 \%$ relative humidity. At the five-leaf stage, robust seedlings with the same size were selected for the following treatments. For Arabidopsis growth, Arabidopsis seeds with or without HT stress were sterilized on the surface and planted on 1/2 Murashige and Skoog (MS) medium and grew under a $14 \mathrm{~h}$ of light $/ 10 \mathrm{~h}$ of dark at $23^{\circ} \mathrm{C}$. Five-day-old Arabidopsis seedlings were used for HT treatment.

\subsection{Expression Analysis of SlCML39}

First, we clarified the SIMPK1-mediated expression of SICML39. The SIMPK1-overexpressing line OE11 and SIMPK1-RNA interference line 1-24 were used in our previous report [26]. The four-week-old seedlings of WT, OE11, and 1-24 were treated with control $\left(25^{\circ} \mathrm{C}\right)$ and HT $\left(42^{\circ} \mathrm{C}\right)$ for $4 \mathrm{~h}$. The leaves were collected for further RNA extraction.

Second, we clarified the organ-specific expression of SlCML39. The ePLANT data of SlCML39 expression were obtained from bar.utoronto.ca (tomato eFP browser). RNA sequence expression data were obtained from different organs of Solanum lycopersicum cv. Heinz. qRT-PCR was used to detect the organ-specific expression of SlCML39. Total RNA was isolated from tomato root, stem, leaf, flower, green fruit, and red fruit.

The four weeks-old tomato seedlings were exposed to drought, salt, $\mathrm{HT}$, cold, $\mathrm{H}_{2} \mathrm{O}_{2}$, and ABA treatments for 1,3,6, 12, and/or $24 \mathrm{~h}$. For salt and drought stresses, the plants were immersed in $100 \mathrm{mM} \mathrm{NaCl}$ and $20 \%$ poly(ethylene glycol)-6000, respectively, for 1 , $3,6,12$, and $24 \mathrm{~h}$. In terms of HT and cold stresses, the seedlings were exposed to HT $\left(42{ }^{\circ} \mathrm{C}\right)$ and cold $\left(4^{\circ} \mathrm{C}\right)$, respectively, for $1,3,6$, and $12 \mathrm{~h}$. Then, $100 \mathrm{uM} \mathrm{ABA}$ and $10 \mathrm{mM}$ $\mathrm{H}_{2} \mathrm{O}_{2}$ were sprayed on tomato leaves for $\mathrm{ABA}$ and $\mathrm{H}_{2} \mathrm{O}_{2}$ treatments, respectively, and the processing time lasted $24 \mathrm{~h}$. The seedlings under normal conditions were used as control. The leaves were collected at the appointed time and preserved at $-70{ }^{\circ} \mathrm{C}$ for further RNA extraction.

\subsection{Bioinformatics Analysis of SlCML39}

The location of SlCML39 in chromosomes was checked in the Tomato ITAG4.0 genome database and the SlCML39 gene structure was confirmed by NCBI and visualized by GSDS 2.0 (Gene Structure Display Server 2.0 (gao-lab.org), accessed on 1 August 2021). Online PlantCARE analyzed the cis-regulatory elements in the $2000 \mathrm{bp}$ promoter region from Transcription Start Site (TSS) of SlCML39 downloaded from ITAG4.0 (Bioinformatics and Systems Biology (ugent.be), accessed on 1 August 2021), and the cis-elements were analyzed by the PlantCARE database (https: / / solgenomics.net, accessed on 1 August 2021), which were visualized on the TBtool software. The MEME tool (MEME Suite (meme-suite.org), accessed on 1 August 2021) was used to predict and analyze the conserved motifs of tomato SICML39. The EF hand motifs were analyzed according to Zhang et al. [20]. The PI and MW 
of SICML39 protein were measured using the online ExPASy tool (https:/ / web.expasy.org, accessed on 1 August 2021).

Sequence alignment of SICML39 with homologous CMLs of Arabidopsis and rice were aligned using MUSCLE [63] and translated by GeneDoc software including Arabidopsis AT5G42380 (AtCML37), AT1G76650 (AtCML38), AT1G76640 (AtCML39), AT3G50770 (ATCML41); Oryza sativa Os01G72550 (OsCML19); and Solanum lycopersicum Solyc11g071740 (SICML39). The phylogenetic tree was constructed using MUSCLE, PhyML, and TreeDyn at www.phylogeny.fr [63]. Along with the six proteins in the above sequence alignment, AT3G01830 (AtCML40), Zea mays (ZmPHB47.K022800.1.p), Solyc102g094000 (S1CML37), and Solanum tuberosum (Soltu.DM.11G024600) were also included.

\subsection{Heterologous Expression of SlCML39 in Arabidopsis}

To construct transgenic Arabidopsis lines overexpressing SICML39, the full-length cDNA fragment of SlCML39 was inserted in the binary vector pBI121 driven by CaMV 35S promoter. Arabidopsis transformation was carried out by the floral dip method. The T1 transformant was screened by detecting the (3:1) isolation rate of kanamycin resistance according to Ding et al. [26]. Two T2 generation homozygote plants (OE4-8 and OE7-9) were obtained, and the expression level of SlCML39 was analyzed by qRT-PCR. The two lines were also used for the study.

\subsection{HT Tolerance of Arabidopsis}

For seed germination and post-germinated growth, dry seeds of WT and SlCML39overexpressing line OE4-8 and OE7-9 were immersed at $52{ }^{\circ} \mathrm{C}$ for 15 (T1) and $25 \mathrm{~min}$ (T2) according to the method of Yokotani et al. [64]. After imbibition, seeds were sterilized on the surface before sowing on $1 / 2$ MS medium. The germination rate and posted-growth phenotype were observed within 8 days of seed germination. For seedling growth, the plates containing 7-day-old seedlings were exposed to $45^{\circ} \mathrm{C}$ for $0 \mathrm{~min}$ (control), 45 (T1), 60 (T2), and 90 (T3). Then, they recovered under standard conditions $\left(23^{\circ} \mathrm{C}\right)$ for 7 days and the plant fresh weight and the chlorophyll (Chl) content were determined. The Chl content in the disc leaves was measured spectrophotometrically, which was carried out after extraction of the leaf discs with $95 \%$ ethanol.

\subsection{Seed Germination under $A B A$ Treatment}

Seeds from Arabidopsis WT and SlCML39-overexpressing line OE4-8 and OE7-9 were sown on $1 / 2 \mathrm{MS}$ medium in the same plates with $0,1.0$, or $1.5 \mu \mathrm{M}$ ABA. Seeds were incubated in the dark at $4{ }^{\circ} \mathrm{C}$ for 3 days to break dormancy, then moved to a growth chamber at $23^{\circ} \mathrm{C}$ under a 16-hour-light/8-hour-dark photoperiod. The germination (radicle emergence) was recorded every day. On the 10th day, photos were taken to show the growth, and the numbers of cotyledon with open and green leaves were also recorded.

\subsection{ABA Content}

Seven-day-old seedlings of SICML39-overexpressing lines OE4-8 and OE7-9 and WT plants were exposed to HT $\left(42^{\circ} \mathrm{C}\right)$ for $2 \mathrm{~h}$. After hormone extraction and solid phase extraction, concentration, and redissolution, $\mathrm{ABA}$ was detected qualitatively and quantitatively by UPLC-ESI-MS/MS according to the method of Chen et al. [65] with some modifications. The samples were analyzed using an ACQUITY UPLC H-Class system (Waters, Milford, CT, USA).

\subsection{RNA-Seq}

According to Lu's experimental design of analyzing HT response by RNA-seq [29], we prepared two separate cDNA libraries: 7-day-old WT plants under HT stress (HT, $2 \mathrm{~h}$ at $\left.42{ }^{\circ} \mathrm{C}\right)$; SlCML39-overexpressing line OE7-9 under HT stress (HT, $2 \mathrm{~h}$ at $\left.42{ }^{\circ} \mathrm{C}\right)$. Each library has 3 replicates, for a total of six samples. The RNA extractions, as well as the quality and quantity measurements, were carried out as previously described by Ding 
et al. [66], and sequenced on a BGISEQ-500 platform at Huada Genomics Institute [28]. After preprocessing and quality control, the clean reads were separated from the raw data. After assembly, according to Langmead and Salzberg [67], by using Bowtie2 (v2.2.5), clean reads were mapped to unigenes. The transcript levels were calculated and normalized to FPKM (fragments per kilobase per transcript per million mapped reads) using RSEM software (v1.2.12) [68]. The distribution statistics of the FPKM of genes were visualized as boxplots using R software (version 3.5.2). The $\mid \log _{2}$ ratio $\mid \geq 1$ and adjusted $p$-value $\leq 0.05$ as the threshold to judge differentially expressed genes (DEGs). GO enrichment analyses were performed using the OmicShare tools (https://www.omicshare.com/tools/, accessed on 10 August 2021).

\section{9. $q R T-P C R$}

For SlCML39 expression, total RNA was extracted from Sections 2.2 and 2.4 above. To verify the accuracy of RNA-seq data, six DEGs were selected. Independent RNA with three replicates of Arabidopsis leaves (WT, OE7-9) under HT was prepared. The TriZol reagent was used for RNA extraction (Takara Bio, Kusatsu shi, Japan). The cDNA Synthesis SuperMix Kit was used to synthesize the first strand of complementary DNA (cDNA) from $1.0 \mu \mathrm{g}$ of total RNA. SYBR Premix Ex Taq (Takara) was used to run qRT-PCR (ABI 7500 Realtime PCR Systems, Thermo Fisher Scientific, Waltham, MA, USA). Triplicate measurements were performed. Tomato UBI and Arabidopsis ACTIN were used as an internal control for tomato and Arabidopsis, respectively. The transcript level was normalized as an internal control. Finally, the relative expression level of each gene was calculated according to the $2^{-\triangle \Delta C T}$ method. Primer sequences are listed in Table S1.

\section{Conclusions}

In conclusion, this study provides a substantial body to support SlCML9 as a stressresponsive gene and its role in the negative regulation of HT tolerance. Overexpression of SlCML39 decreased seed germination and seedling growth under HT stress. Our data propose a mechanism whereby the stress/ABA-responsive genes are regulated by HT through SICML39. A simplified working model is shown in Figure 10, which proposes a new pathway combined with the complex ABA and HT response networks in plants. These findings also provide an exciting opportunity for the exploitation of SlCML39 as a potential modulator for plant HT tolerance as well as abiotic stresses.

Supplementary Materials: The following are available online at https:/ / www.mdpi.com/article/10 $.3390 /$ ijms222111479/s1.

Author Contributions: H.D. conceived of the work. H.D. and Y.Q. performed the experiment contents. Y.F., Y.J., and J.S. assisted in the experiments. H.D. wrote the manuscript. C.G. supervised the writing. All authors have read and agreed to the published version of the manuscript.

Funding: This work was supported by the Natural Science Foundation of Jiangsu Province (BK20191437), the National Natural Science Foundation of China (grant No. 32172546), and the China Postdoctoral Fund (2018M630611).

Institutional Review Board Statement: Not applicable.

Informed Consent Statement: Not applicable.

Data Availability Statement: The data presented in this study are available in the article and Supplementary Materials.

Conflicts of Interest: The authors declare that they have no competing interests.

\section{References}

1. Kudla, J.; Becker, D.; Grill, E.; Hedrich, R.; Hippler, M.; Kummer, U.; Parniske, M.; Romeis, T.; Schumacher, K. Advances and current challenges in calcium signaling. New Phytol. 2018, 218, 414-431. [CrossRef] [PubMed]

2. Zhu, X.; Dunand, C.; Snedden, W.; Galaud, J. CaM and CML emergence in the green lineage. Trends Plant Sci. 2015, 20, 483-489. [CrossRef] 
3. DeFalco, T.A.; Bender, K.W.; Snedden, W.A. Breaking the code: $\mathrm{Ca}^{2+}$ sensors in plant signal. Biochem. J. 2010, 425, 27-40. [CrossRef] [PubMed]

4. Gifford, J.L.; Walsh, M.P.; Vogel, H.J. Structures and metal-ion-binding properties of the $\mathrm{Ca}^{2+}$-binding helix-loop-helix EF-hand motifs. Biochem. J. 2007, 405, 199-221. [CrossRef] [PubMed]

5. Mohanta, T.K.; Kumar, P.; Bae, H. Genomics and evolutionary aspect of calcium signaling event in calmodulin and calmodulin-like proteins in plants. BMC Plant Biol. 2017, 17, 38. [CrossRef]

6. McCormack, E.; Braam, J. Calmodulins and related potential calcium sensors of Arabidopsis. New Phytol. 2003, 159, 585-598. [CrossRef]

7. He, X.; Liu, W.; Li, W.; Liu, Y.; Wang, W.; Xie, P.; Kang, Y.; Liao, L.; Qian, L.; Liu, Z.; et al. Genome-wide identification and expression analysis of cam/cml genes in brassica napus under abiotic stress. J. Plant Physiol. 2020, 255, 153251. [CrossRef] [PubMed]

8. Tang, M.; Xu, C.; Cao, H.; Shi, Y.; Chen, J.; Chai, Y.; Li, Z. Tomato calmodulin-like protein SlCML37 is a calcium (Ca $\left.{ }^{2+}\right)$ sensor that interacts with proteasome maturation factor SIUMP1 and plays a role in tomato fruit chilling stress tolerance. J. Plant Physiol. 2021, 25, 153373. [CrossRef] [PubMed]

9. Yang, X.; Wang, S.S.; Wang, M.; Qiao, Z.; Bao, C.C.; Zhang, W. Arabidopsis thaliana, calmodulin-like protein CML24 regulates pollen tube growth by modulating the actin cytoskeleton and controlling the cytosolic $\mathrm{Ca}^{2+}$ concentration. Plant Mol. Biol. 2014, 86, 225-236. [CrossRef]

10. Wang, S.S.; Diao, W.Z.; Yang, X.; Qiao, Z.; Wang, M.; Acharya, B.R.; Zhang, W. Arabidopsis thaliana CML25 mediates the $\mathrm{Ca}^{2+}$ regulation of $\mathrm{K}^{+}$transmembrane trafficking during pollen germination and tube elongation. Plant Cell Environ. 2015, 38, 2372-2386. [CrossRef]

11. Bender, K.W.; Rosenbaum, D.M.; Vanderbeld, B.; Ubaid, M.; Snedden, W.A. The Arabidopsis calmodulin-like protein, CML39, functions during early seedling establishment. Plant J. 2013, 76, 634-647. [CrossRef]

12. Dobney, S.; Chiasson, D.; Lam, P.; Smith, S.P.; Snedden, W.A. The calmodulin-related calcium sensor CML42 plays a role in trichome branching. J. Biol. Chem. 2009, 284, 31647-31657. [CrossRef] [PubMed]

13. Vadassery, J.; Reichelt, M.; Hause, B.; Gershenzon, J.; Boland, W.; Mithofer, A. CML42-mediated calcium signaling coordinates responses to Spodoptera herbivory and abiotic stresses in Arabidopsis. Plant. Physiol. 2012, 159, 1159-1175. [CrossRef]

14. Xu, B.; Cheval, C.; Laohavisit, A.; Hocking, B.; Chiasson, D.; Olsson, T.S.G.; Shirasu, K.; Faulkner, C.; Gilliham, M. A calmodulinlike protein regulates plasmodesmal closure during bacterial immune responses. New Phytol. 2017, 215, 77-84. [CrossRef] [PubMed]

15. Astegno, A.; Bonza, M.C.; Vallone, R.; La Verde, V.; D'Onofrio, M.; Luoni, L.; Molesini, B.; Dominici, P. Arabidopsis calmodulinlike protein CML36 is a calcium $\left(\mathrm{Ca}^{2+}\right)$ sensor that interacts with the plasma membrane $\mathrm{Ca}^{2+}$-ATPase isoform ACA8 and stimulates its activity. J. Biol. Chem. 2017, 292, 15049-15061. [CrossRef]

16. Magnan, F.; Ranty, B.; Charpenteau, M.; Sotta, B.; Galaud, J.P.; Aldon, D. Mutations in AtCML9, a calmodulin-like protein from Arabidopsis thaliana, alter plant responses to abiotic stress and abscisic acid. Plant J. 2008, 56, 575-589. [CrossRef] [PubMed]

17. Xu, G.Y.; Rocha, P.S.; Wang, M.L.; Xu, M.L.; Cui, Y.C.; Li, L.Y.; Zhu, Y.X.; Xia, X. A novel rice calmodulin-like gene, OsMSR2, enhances drought and salt tolerance and increases ABA sensitivity in Arabidopsis. Planta 2011, 234, 47-59. [CrossRef]

18. Chen, C.; Sun, X.; Duanmu, H.; Zhu, D.; Yu, Y.; Cao, L.; Liu, A.; Jia, B.; Xiao, J.; Zhu, Y. GsCML27, a gene encoding a calcium binding EF-hand protein from Glycine soja, plays differential roles in plant responses to Bicarbonate, salt and osmotic stresses. PLoS ONE 2015, 10, e0141888. [CrossRef]

19. Wu, X.; Qiao, Z.; Liu, H.; Acharya, B.R.; Li, C.; Zhang, W. CML20, an arabidopsis calmodulin-like protein, negatively regulates guard cell aba signal and drought stress tolerance. Front. Plant Sci. 2017, 8, 824. [CrossRef]

20. Zhang, Y.; Huang, J.; Hou, Q.; Liu, Y.; Wang, J.; Deng, S. Isolation and functional characterization of a Salt-responsive calmodulinlike gene MpCML40 from semi-mangrove Millettia pinnata. Int. J. Mol. Sci. 2021, 22, 3475. [CrossRef]

21. Munir, S.; Liu, H.; Xing, Y.; Hussain, S.; Ouyang, B.; Zhang, Y.; Li, H.; Ye, Z. Overexpression of calmodulin-like (ShCML44) stress-responsive gene from Solanum habrochaites enhances tolerance to multiple abiotic stresses. Sci. Rep. 2016, 6, 1-20. [CrossRef]

22. Shi, J.; Du, X. Identification, characterization and expression analysis of calmodulin and calmodulin-like proteins in Solanum pennellii. Sci Rep. 2020, 10, 1-17. [CrossRef] [PubMed]

23. Chaisson, D.; Ekengren, S.K.; Martin, G.B.; Dobney, S.L.; Snedden, W.A. Calmodulin-like proteins from Arabidopsis and tomato are involved in host defense against Pseudomonas syringae pv. tomato. Plant Mol. Biol. 2005, 58, 887-897. [CrossRef] [PubMed]

24. Ding, H.; Mo, S.; Qian, Y.; Yuan, G.; Wu, X.; Ge, C. Integrated proteome and transcriptome analyses revealed key factors involved in tomato (Solanum lycopersicum) under high temperature stress. Food Energy Sec. 2020, 9, e239. [CrossRef]

25. Munir, S.; Khan, M.R.; Song, J.; Munir, S.; Zhang, Y.; Ye, Z.; Wang, T. Genome wide identification, characterization and expression analysis of calmodulin-like (CML) proteins in tomato (Solanum lycopersicum). Plant Physiol. Biochem. 2016, 102, 167-179. [CrossRef]

26. Ding, H.; He, J.; Wu, Y.; Wu, X.; Ge, C.; Wang, Y.; Zhong, S.; Peiter, E.; Liang, J.; Xu, W. The tomato mitogen-activated protein kinase SIMPK1 is as a negative regulator of the high temperature. Plant Physiol. 2018, 177, 633-651. [CrossRef]

27. Lu, P.P.; Zheng, W.J.; Wang, C.T.; Shi, W.; Fu, J.; Chen, M.; Chen, J.; Zhou, Y.; Xi, Y.; Xu, Z. Wheat Bax Inhibitor-1 interacts with TaFKBP62 and mediates response to heat stress. BMC Plant. Bio. 2018, 18, 259. [CrossRef] 
28. Zhu, F.Y.; Chen, M.X.; Ye, N.H.; Qiao, W.M.; Gao, B.; Law, W.K.; Tian, Y.; Zhang, D.; Zhang, D.; Liu, T.Y.; et al. Comparative performance of the BGISEQ-500 and Illumina HiSeq4000 sequencing platforms for transcriptome analysis in plants. Plant Methods 2018, 14, 69. [CrossRef]

29. Chao, L.M.; Liu, Y.Q.; Chen, D.Y.; Xue, X.Y.; Mao, Y.B.; Chen, X.Y. Arabidopsis transcription factors SPL1 and SPL12 confer plant thermotolerance at reproductive stage. Mol. Plant 2017, 10, 735-748. [CrossRef] [PubMed]

30. Nguyen, A.H.; Matsui, A.; Tanaka, M.; Mizunashi, K.; Nakaminami, K.; Hayashi, M.; Iida, K.; Toyoda, T.; Nguyen, D.V.; Seki, M. Loss of Arabidopsis 5' $5^{\prime}$ exoribonuclease AtXRN4 function enhances heat stress tolerance of plants subjected to severe heat stress. Plant Cell Physiol. 2015, 56, 1762-1772. [CrossRef] [PubMed]

31. Hu, Z.; Song, N.; Zheng, M.; Liu, X.; Liu, Z.; Xing, J.; Ma, J.; Guo, W.; Yao, Y.; Peng, H.; et al. Histone acetyltransferase GCN 5 is essential for heat stress-responsive gene activation and thermotolerance in Arabidopsis. Plant J. 2015, 84, 1178-1191. [CrossRef] [PubMed]

32. Wiese, A.J.; Steinbachová, L.; Timofejeva, L.; Čermák, V.; Klodová, B.; Ganji, R.S.; Limones-Mendez, M.; Bokvaj, P.; Hafidh, S.; Potešil, D.; et al. Arabidopsis bZIP18 and bZIP52 accumulate in nuclei following heat stress where they regulate the expression of a similar set of genes. Int. J. Mol. Sci. 2021, 22, 530. [CrossRef]

33. Weng, J.K.; Ye, M.; Li, B.; Noel, J.P. Co-evolution of hormone metabolism and signaling networks expands plant adaptive plasticity. Cell 2016, 166, 881-893. [CrossRef]

34. Zhu, Y.; Wang, B.; Tang, K.; Hsu, C.C.; Xie, S.; Du, H.; Yang, Y.; Tao, W.A.; Zhu, J.K. An arabidopsis nucleoporin NUP85 modulates plant responses to ABA and salt stress. PLoS Genet. 2017, 13, e1007124. [CrossRef] [PubMed]

35. Vishwakarma, K.; Upadhyay, N.; Kumar, N.; Yadav, G.; Singh, J.; Mishra, R.K.; Kumar, V.; Verma, R.; Upadhyay, R.G.; Pandey, M.; et al. Abscisic acid signaling and abiotic stress tolerance in plants: A review on current knowledge and future prospects. Front. Plant Sci. 2017, 8, 161. [CrossRef] [PubMed]

36. Ma, Y.; Cao, J.; He, J.; Chen, Q.; Li, X.; Yang, Y. Molecular mechanism for the regulation of ABA homeostasis during plant development and stress responses. Int. J. Mol. Sci. 2018, 19, 3643. [CrossRef]

37. Huang, Y.C.; Niu, C.Y.; Yang, C.R.; Jinn, T.L. The heat stress factor HSFA6b connects ABA signaling and ABA-mediated heat responses. Plant Physiol. 2016, 172, 1182-1199. [CrossRef]

38. Suzuki, N.; Bassil, E.; Hamilton, J.S.; Inupakutika, M.A.; Zandalinas, S.I.; Tripathy, D.; Luo, Y.; Dion, E.; Fukui, G.; Kumazaki, A.; et al. ABA is required for plant acclimation to a combination of salt and heat stress. PLoS ONE 2016, 11, e0147625. [CrossRef]

39. Mo, S.; Qian, Y.; Zhang, W.; Qian, L.; Wang, Y.; Cailin, G.; Ding, H. Mitogen-activated protein kinase action in plant response to high-temperature stress: A mini review. Protoplasma 2021, 258, 477-482. [CrossRef]

40. Farooq, M.A.; Niazi, A.K.; Akhtar, J.; Farooq, M.; Souri, Z.; Karimi, N.; Rengel, Z. Acquiring control: The evolution of ROS-Induced oxidative stress and redox signal pathways in plant stress responses. Plant Physiol. Bioch. 2019, 141, 353-369. [CrossRef]

41. Vanderbeld, B.; Snedden, W.A. Developmental and stimulus-induced expression patterns of Arabidopsis calmodulin-like genes CML37, CML38 and CML39. Plant Mol. Biol. 2007, 64, 683-697. [CrossRef] [PubMed]

42. Scholz, S.S.; Reichelt, M.; Vadassery, J.; Mithöfer, A. Calmodulin-like protein CML37 is a positive regulator of ABA during drought stress in Arabidopsis. Plant Signal. Behav. 2015, 10, e1011951. [CrossRef] [PubMed]

43. Lokdarshi, A.; Conner, W.C.; McClintock, C.; Li, T.; Roberts, D.M. Arabidopsis CML38, a calcium sensor that localizes to ribonucleoprotein complexes under hypoxia stress. Plant Physiol. 2016, 170, 1046-1059. [CrossRef]

44. Campos, W.F.; Dressano, K.; Ceciliato, P.H.; Guerrero-Abad, J.C.; Silva, A.L.; Fiori, C.S.; Amanda, M.D.C.; Bergonci, T.; Claus, L.A.N.; Silva-Filho, M.C.; et al. Arabidopsis thaliana rapid alkalinization factor 1-mediated root growth inhibition is dependent on calmodulin-like protein 38. J. Biol. Chem. 2018, 293, 2159-2171. [CrossRef]

45. Song, X.; Li, J.; Lyu, M.; Kong, X.; Hu, S.; Song, Q.; Zuo, K. CALMODULIN-LIKE-38 and PEP1 RECEPTOR 2 integrate nitrate and brassinosteroid signals to regulate root growth. Plant Physiol. 2021. [CrossRef]

46. Midhat, U.; Ting, M.K.; Teresinski, H.J.; Snedden, W.A. The calmodulin-like protein, CML39, is involved in regulating seed development, germination, and fruit development in Arabidopsis. Plant Mol. Biol. 2018, 96, 375-392. [CrossRef]

47. Zeng, H.; Xu, L.; Singh, A.; Wang, H.; Du, L.; Poovaiah, B.W. Involvement of calmodulin and calmodulin-like proteins in plant responses to abiotic stresses. Front. Plant Sci. 2015, 6, 600. [CrossRef]

48. Dubrovina, A.S.; Aleynova, O.A.; Ogneva, Z.V.; Suprun, A.R.; Ananev, A.A.; Kiselev, K.V. The effect of abiotic stress conditions on expression of calmodulin (CaM) and calmodulin-like (CML) genes in wild-growing grapevine Vitis amurensis. Plants 2019, 8, 602. [CrossRef]

49. Zhao, K.; Shen, X.J.; Yuan, H.Z.; Liu, Y.; Liao, X.; Wang, Q.; Liu, L.L.; Li, F.; Li, T.H. Isolation and characterization of dehydrationresponsive element-binding factor 2C (MsDREB2C) from Malus sieversii Roem. Plant Cell Physiol. 2013, 54, 1415-1430. [CrossRef] [PubMed]

50. Abe, H.; Yamaguchi-Shinozaki, K.; Urao, T.; Iwasaki, T.; Hosokawa, D.; Shinozaki, K. Role of Arabidopsis MYC and MYB homologs in drought- and abscisic acid-regulated gene expression. Plant Cell 1997, 9, 1859-1868.

51. Delk, N.A.; Johnson, K.A.; Chowdhury, N.I.; Braam, J. CML24, regulated in expression by diverse stimuli, encodes a potential $\mathrm{Ca}^{2+}$ sensor that functions in responses to abscisic acid, daylength, and ion stress. Plant Physiol. 2005, 139, 240-253. [CrossRef]

52. Sreenivasulu, N.; Harshavardhan, V.T.; Govind, G.; Seiler, C.; Kohli, A. Contrapuntal role of ABA: Does it mediate stress tolerance or plant growth retardation under long-term drought stress? Gene 2012, 506, 265-273. [CrossRef] [PubMed] 
53. Geng, X.; Zang, X.; Li, H.; Liu, Z.; Zhao, A.; Liu, J.; Peng, H.; Yao, Y.; Hu, Z.; Ni, Z. Unconventional splicing of wheat TabZIP60 confers heat tolerance in transgenic Arabidopsis. Plant Sci. 2018, 274, 252-260. [CrossRef] [PubMed]

54. Brinker, M.; Brosché, M.; Vinocur, B.; Abo-Ogiala, A.; Fayyaz, P.; Janz, D.; Ottow, E.A.; Cullmann, A.D.; Saborowski, J.; Kangasjärvi, J.; et al. Linking the salt transcriptome with physiological responses of a salt-resistant Populus species as a strategy to identify genes important for stress acclimation. Plant Physiol. 2010, 154, 1697-1709. [CrossRef]

55. Koops, P.; Pelser, S.; Ignatz, M.; Klose, C.; Marrocco-Selden, K.; Kretsch, T. EDL3 is an F-box protein involved in the regulation of abscisic acid signal in Arabidopsis thaliana. J. Exp. Bot. 2011, 62, 5547-5560. [CrossRef]

56. Ali, M.A.; Plattner, S.; Radakovic, Z.; Wieczorek, K.; Elashry, A.; Grundler, F.M.; Ammelburg, M.; Siddique, S.; Bohlmann, H. An Arabidopsis ATPase gene involved in nematode-induced syncytium development and abiotic stress responses. Plant J. 2013, 74, 852-866. [CrossRef]

57. Hsu, K.H.; Liu, C.C.; Wu, S.J.; Ku, O.Y.Y.; Lu, C.A.; Wu, C.R.; Lian, P.J.; Hong, C.Y.; Ke, Y.T.; Huang, J.H. Expression of a gene encoding a rice RING zinc-finger protein, OsRZFP34, enhances stomata opening. Plant Mol. Biol. 2014, 86, 125-137. [CrossRef] [PubMed]

58. Wang, R.; Zhang, Y.; Kieffer, M.; Yu, H.; Kepinski, S.; Estelle, M. HSP90 regulates temperature-dependent seedling growth in Arabidopsis by stabilizing the auxin co-receptor F-box protein TIR1. Nat. Commun. 2016, 7, 10269. [CrossRef]

59. Fujita, M.; Fujita, Y.; Maruyama, K.; Seki, M.; Hiratsu, K.; Ohme-Takagi, M.; Tran, L.; Yamaguchi-Shinozaki, K.; Shinozaki, K. A dehydration-induced NAC protein, RD26, is involved in a novel ABA-dependent stress-signaling pathway. Plant J. 2004, 39, 863-876. [CrossRef] [PubMed]

60. Yu, J.; Kang, L.; Li, Y.; Wu, C.; Zheng, C.; Liu, P.; Huang, J. RING finger protein RGLG1 and RGLG2 negatively modulate MAPKKK18 mediated drought stress tolerance in Arabidopsis. J. Integr. Plant Biol. 2021, 63, 484-493. [CrossRef]

61. Li, K.; Yang, F.; Zhang, G.; Song, S.; Li, Y.; Ren, D.; Miao, Y.; Song, C.P. AIK1, a mitogen-activated protein kinase, modulates abscisic acid responses through the MKK5-MPK6 kinase cascade. Plant Physiol. 2017, 173, 1391-1408. [CrossRef] [PubMed]

62. Miles, G.P.; Samuel, M.A.; Ellis, B.E. Suppression of MKK5 reduces ozone-induced signal transmission to both MPK3 and MPK6 and confers increased ozone sensitivity in Arabidopsis thaliana. Plant Signal. Behav. 2009, 4, 687-692. [CrossRef]

63. Dereeper, A.; Guignon, V.; Blanc, G.; Audic, S.; Buffet, S.; Chevenet, F.; Dufayard, J.F.; Guindon, S.; Lefort, V.; Lescot, M.; et al. Phylogeny. fr: Robust phylogenetic analysis for the non-specialist. Nucleic Acids Res. 2008, 36, 465-469. [CrossRef] [PubMed]

64. Yokotani, N.; Ichikawa, T.; Kondou, Y.; Matsui, M.; Hirochika, H.; Iwabuchi, M.; Oda, K. Expression of rice heat stress transcription factor OsHsfA2e enhances tolerance to environmental stresses in transgenic Arabidopsis. Planta 2008, 227, 957-967. [CrossRef] [PubMed]

65. Chen, M.L.; Fu, X.M.; Liu, J.Q.; Ye, T.T.; Hou, S.Y.; Huang, Y.Q.; Yuan, B.F.; Wu, Y.; Feng, Y.Q. Highly sensitive and quantitative profiling of acidic phytohormones using derivatization approach coupled with nano-LC-ESI-Q-TOF-MS analysis. J. Chromatogr. B Anal. Technol. Biomed. Life Sci. 2012, 905, 67-74. [CrossRef]

66. Ding, H.; Yuan, G.; Mo, S.; Qian, Y.; Wu, Y.; Chen, Q.; Xu, X.X.; Wu, X.X.; Ge, C. Genome-wide analysis of the plant-specific VQ motif-containing proteins in tomato (Solanum lycopersicum) and characterization of SIVQ6 in thermotolerance. Plant Physiol. Biochem. 2019, 143, 29-39. [CrossRef] [PubMed]

67. Langmead, B.; Salzberg, S.L. Fast gapped-read alignment with Bowtie 2. Nat. Methods 2012, 9, 357-359. [CrossRef]

68. Li, B.; Dewey, C.N. RSEM: Accurate transcript quantification from RNA-Seq data with or without a reference genome. BMC Bioinform. 2011, 12, 323. [CrossRef] 